\title{
A method for Precise Point Positioning with decimetre accuracy using Wide-Lane Ambiguities and Triple-frequency GNSS Data
}

\author{
Manoj Deo \& Ahmed El-Mowafy \\ School of Earth and Planetary Sciences \\ Curtin University, Bently \\ WA 6102, Australia \\ Email: manoj.deo01@gmail.com
}

\begin{abstract}
This paper proposes a precise point positioning (PPP) approach that offers an accuracy of a few centimetres $(\mathrm{cm})$ with triple frequency GNSS data. Firstly, an enhanced triple frequency linear combination is presented for rapid fixing of the extra wide-lane (EWL) and wide-lane (WL) ambiguities for GPS, Beidou Phase II and Galileo. This has improved performance compared to the Melbourne-Wübbena (MW) linear combination, and has $6.7 \%$ lower measurement noise for the GPS L1/L2 signals, $12.7 \%$ for L1/L5 and 0.7\% for L2/L5. Analysis with tested data showed a $5.4 \%$ and $5.9 \%$ reduction in time required to fix the $\mathrm{N}_{21}$ and $\mathrm{N}_{51}$ ambiguities, respectively.
\end{abstract}

Once the EWL/ WL ambiguities are fixed with the proposed linear combinations, three methods are presented that aim to provide decimetre $(\mathrm{dm})$ level positioning accuracy. In the first approach, the three EWL/ WL ambiguities are used in their respective carrier phase only WL equations to derive a low-noise ionosphere-free (IF) linear combination. The second method uses a low noise IF combination with two of the carrier-phase EWL/WL combinations and pseudorange measurement on a single frequency, whereas the third method uses a low noise IF combination with a single carrier phase EWL combination and pseudorange measurements on two frequencies. These proposed methods can provide dm level positioning accuracy if carrier phase measurements with $\mathrm{mm}$ precision is tracked by the receiver. When comparing these combinations with a combination proposed in Li et al. (2014), it is found that superior performance is achieved particularly with the third method in high carrier phase multipath environment. The advantage of these models compared with the traditional PPP that needs 30 minutes on average to converge to the dm positional accuracy is that the same accuracy or better can be achieved instantly after the EWL/ WL ambiguities are resolved within a few epochs. It is also useful for environments with high multipath and noise.

Keywords: precise point positioning, linear combinations, GNSS

\section{Introduction}

Precise point positioning (PPP) (Zumberge et al., 1997) is a well-established technique for achieving centimetre $(\mathrm{cm})$ level accuracy for a static stand-alone GNSS receiver, and decimetre $(\mathrm{dm})$ level accuracy for a kinematic positioning. Since it provides consistent performance on a global scale, it is well suited for applications with limited geodetic infrastructure and at remote locations (Bisnath et al., 2018). Dual frequency PPP models have been developed that use either a float ambiguity estimation (Zumberge et al., 1997; Heroux and Kouba, 2001) or integer ambiguity resolution (PPP-AR) approach (Ge et al., 2008; Geng et al. 2010; Laurichesse et al., 2009; Collins, 2010). However, a drawback of both float-PPP and PPP-AR methods is a long period of several tens of minutes required for the float ambiguities to converge at $\mathrm{cm}$ level precision, or to fix the integer ambiguities in the case of PPP-AR. This is due to the use of high noise pseudorange measurements that can only be reduced over time averaging, accompanied 
by a change in satellite geometry (El-Mowafy 2014) as well as multipath, satellite geometry and quality of measurements (Bisnath et al., 2009).

PPP-AR models require satellite Uncalibrated Phase Delay (UPD) to separate the float carrier phase ambiguities from their integer values and fix the latter. There are three main PPP-AR methods that use ionosphere-free (IF) implementations, namely (1) Fractional Cycle Bias (FCB) proposed in Ge et al. (2008), (2) Integer Recovery Clock (IRC) proposed in Laurichesse et al. (2009) and (3) Decoupled Satellite Clock (DSC) proposed by Collins (2010). Teunissen and Khodabandeh (2014) proposed PPP-RTK models based on S-System theory for a comon clock and distinct clock parametrisation. The FCB, IRC and DSC models were shown to be intristically the same and differ due to (a) the choice of S-basis, (b) the choice of parametrisation, and (c) the choice of whether or not to eliminate the ionosphere (Teunissen and Khodabandeh, 2014).

The procedure for PPP-AR starts with resolution of the extra wide-lane (EWL) and wide-lane (WL) ambiguity, followed by fixing the narrow-lane (NL) ambiguity, which has a shorter wavelength than any of the individual carrier phase wavelengths ( $\mathrm{Li}, 2018)$. NL ambiguity resolution is comparatively more challenging than the EWL/WL ambiguities, due to the short wavelength involved. This is true even in the double differenced relative positioning model ( $\mathrm{Li}$ et al., 2010). Although tripple frequency PPP-AR models follow the three carrier ambiguity resolution (TCAR) approach (Vollath et al. 1998; Jung et al. 2000) that is typically used in relative positioning models, they use IF implementations. Geng and Bock (2013) presented a PPP-AR approach for the triple-frequency GPS case, where the L2/L5 EWL ambiguity was firstly resolved using the Melbourne-Wübbena (MW) combination. This was then expressed in terms of L1/L2 WL carrier phase measurements to form a carrier phase only IF observable with a wavelength of $3.4 \mathrm{~m}$. This was subsequently used with the L1/L2 dual-frequency IF pseudorange only linear combination to fix the L1/L2 WL ambiguity. Once the WL ambiguity was fixed, it was used to resolve the NL ambiguity. Using simulated data, the authors of that study showed that the NL ambiguity can be resolved correctly with $99 \%$ in just over a minute with carrier phase precision of $1-2 \mathrm{~mm}$. Li et al. (2014) enhanced the step for estimating the WL ambiguity by using a triple frequency linear combination of pseudorange measurements with noise minimisation, IF and geometry preserving properties.

Triple frequency data also improves PPP models with float ambiguity estimation. Such models take advantage of the triple-frequency data to form linear combinations that improve the performance. Triple-frequency linear combinations with integer coefficients were suggested in Cocard et al. (2008) and Urquhart (2009). However, these linear combinations are suited for relative positioning models since they were not totally ionosphere free. A triple frequency lownoise and ionosphere free phase only linear combination were proposed by Li et al. (2014) and Deo and El-Mowafy (2016). In the latter paper, a mixed code-phase linear combination was also proposed with low noise and IF properties. These carrier phase combinations contain the individual carrier phase ambiguities or NL ambiguities which require several tens of minutes to converge at $\mathrm{cm}$ level precision, thus is not ideal for real time applications.

This paper firstly proposes an enhanced triple frequency linear combination for faster fixing of the EWL/ WL ambiguities compared to the commonly used MW combination. Compared to MW, these combinations have lower measurement noise relative to the wavelength, and this enables faster EWL/WL ambiguity estimation. Secondly this paper presents a PPP approach which provides dm level positioning accuracy instantly after the EWL/WL ambiguities are correctly estimated, which typically takes 1-2 minutes. Hence, users avoid the lengthy 
convergence time required to estimate float ambiguities, or fix the NL integer ambiguities in the case of PPP-AR. Three PPP models are proposed that give dm level accuracy instantly after the EWL/ WL ambiguities are fixed. The first method is based on an IF linear combination from the three EWL/ WL phase equations, with minimum measurement noise propagation. The second method uses two of the three EWL/ WL phase equations and an individual pseudorange measurement to form an IF low noise combination, whereas the third method uses a single EWL/ WL phase equation and pseudorange measurement on two frequencies to form an IF low noise combination.

The next section presents the GNSS observation equations, followed by the development of improved linear combinations that enable enhanced fixing of the EWL and WL ambiguities. Section 4 presents the three models for dm level precise positioning after fixing the EWL and WL ambiguities. Section 5 presents analysis and validation of these methods, followed by the conclusions.

\section{The GNSS Observation Equations}

The observation equations for the pseudorange and carrier phase measurements for frequency $j$ and satellite $k$ from a GNSS constellation, such as GPS (denoted here as $G$ ), for one receiver are:

$$
\begin{gathered}
P_{j}^{k_{G}}=\rho^{k_{G}}+c\left(d t_{\mathrm{G}}-d t^{k_{G}}+d_{j_{G}}-d_{j}{ }^{k_{G}}\right)+T^{k_{G}}+\frac{f_{1}^{2}}{f_{j}^{2}} I^{k_{G}}+\varepsilon_{P_{j}}^{k_{G}} \\
\Phi_{j}^{k_{G}}=\rho^{k_{G}}+c\left(d t_{G}-d t^{k_{G}}+\delta_{j_{G}}-\delta_{j}^{k_{G}}\right)+T^{k_{G}}-\frac{f_{1}^{2}}{f_{j}^{2}} I^{k_{G}}+\lambda_{j} N_{j}^{* k_{G}}+\varepsilon_{\Phi_{j}}^{k_{G}}
\end{gathered}
$$

where $P_{j}$ is the pseudorange and $\Phi_{j}$ is the carrier phase measurement in distance units; $f_{j}$ is the transmitting frequency; $\rho^{k_{G}}$ is the satellite-to-receiver geometric range; $c$ is the speed of light in vacuum; $d t_{G}$ and $d t^{k_{G}}$ are the receiver and satellite clock offsets, where the latter is eliminated in PPP by the use of precise clock corrections; $T^{k_{G}}$ is the tropospheric delay; $\lambda_{j}$ denotes the wavelength for frequency $j ; I^{k_{G}}$ is the ionosphere error for a reference frequency, e.g. $i=\mathrm{L} 1$ for GPS; $\varepsilon_{\Phi_{j}}^{k_{G}}$ and $\varepsilon_{P_{j}}^{k_{G}}$ include measurement noise and multipath of the phase and code measurements, respectively. $d_{j_{G}}$ is the receiver hardware bias for code measurements on frequency $j$. Similarly, $d_{j}{ }^{k_{G}}$ is the satellite hardware bias which also includes the satellite Differential Code Biases (DCB) of a reference IF combination (i.e. L1/L2 pseudoranges for GPS) that exists because of the use of satellite clock offsets determined from IF measurements with embedded DCBs. Likewise, $\delta_{j_{G}}$ and $\delta_{j}{ }^{k_{G}}$ are the receiver and satellite hardware biases for the carrier phase measurements. $N_{j}^{* k_{G}}$ is the non-integer ambiguity term containing the fractional phase biases in the receiver and the satellite hardware.

It is assumed that users have applied the satellite carrier phase and code biases available apriori from external sources and performed between-satellite-single-differencing (BSSD) of measurements to remove receiver hardware biases and clock error. Also, when integrating measurements from several constellations, additional biases such as inter-system biases (ISBs) and inter-system satellite time offsets must be considered (El-Mowafy et al., 2016). In the following sections, we assume the same measurement precision denoted by standard deviations 
(std) for all carrier phase measurements and for all available triple frequencies $i, j$ and $k$, and the same for code measurements. Thus, $\sigma_{\Phi}=\sigma_{\Phi_{i}}=\sigma_{\Phi_{j}}=\sigma_{\Phi_{k}}$ and $\sigma_{\mathrm{P}}=\sigma_{\mathrm{P}_{i}}=\sigma_{\mathrm{P}_{j}}=\sigma_{\mathrm{P}_{k}}$.

\section{Improved Linear Combination for EWL and WL Ambiguity Estimation}

This section, presents the development of an enhanced code-carrier combination which preserves the EWL/ WL ambiguity, but has lower measurement noise than MW. For a user accessing triple-frequency carrier phase and code measurements, a linear combination is formed as follows:

$$
\psi_{i j}=\frac{c}{f_{i}-f_{j}}\left(\phi_{i}-\phi_{j}\right)+\alpha_{i j} P_{i}+\beta_{i j} P_{j}+\gamma_{i j} P_{k}
$$

where $\phi_{i}$ and $\phi_{j}$ are the carrier phase measurements in cycle units for frequencies $i$ and $j$, respectively; $P_{i}, P_{j}$ and $P_{k}$ are the code measurements in distance units; $\alpha_{i j}, \beta_{i j}$ and $\gamma_{i j}$ are the combination coefficients associated with frequencies $i$ and $j$, applied for code measurements; and $f_{i}, f_{j}$ are the transmitting frequencies. The coefficient $\frac{\mathrm{c}}{\mathrm{f}_{\mathrm{i}}-\mathrm{f}_{\mathrm{j}}}$ ensures that a WL ambiguity persists in this combination, whereas the pseudorange coefficients for the three frequencies enable reduction of the overall measurement noise. The satellite and receiver identifiers have been removed for simplicity since the equation is concerned with a single receiver and one satellite. A similar equation may be formed for carrier frequencies $i$ and $k$ as

$$
\psi_{i k}=\frac{c}{f_{i}-f_{k}}\left(\phi_{i}-\phi_{k}\right)+\alpha_{i k} P_{i}+\beta_{i k} P_{j}+\gamma_{i k} P_{k}
$$

where $\phi_{k}$ is the carrier phase measurement for frequency $k$ on transmitting frequency $f_{k}$. The carrier phase coefficients in cycle units of +1 and -1 ensure that the WL phase ambiguity is preserved. When expressing phase measurements in distance units $\left(\Phi_{i}=\lambda_{i} \phi_{i}, \Phi_{j}=\lambda_{j} \phi_{j}\right)$, Eqn. 3 becomes:

$$
\Psi_{i j}=\frac{f_{i}}{f_{i}-f_{j}} \Phi_{i}-\frac{f_{j}}{f_{i}-f_{j}} \Phi_{j}+\alpha_{i j} P_{i}+\beta_{i j} P_{j}+\gamma_{i j} P_{k}
$$

In determining the coefficients $\alpha_{i j}, \beta_{i j}$ and $\gamma_{i j}$, three conditions are placed such that the combination is geometry-free, first order IF and has least measurement noise propagation. The resulting combination replaces the MW combination with the advantage of lower measurement noise, hence enabling faster ambiguity resolution. The first order IF condition is achieved by equating the sum of the ionospheric terms in Eqn. 5 to zero as

$$
\frac{f_{i}}{f_{i}-f_{j}} \frac{f_{1}^{2}}{f_{i}^{2}} I+\frac{f_{j}}{f_{i}-f_{j}} \frac{f_{1}^{2}}{f_{j}^{2}} I+\alpha_{i j} I+\beta_{i j} \frac{f_{1}^{2}}{f_{j}^{2}} I+\gamma_{i j} \frac{f_{1}^{2}}{f_{k}^{2}} I=0
$$

which is rearranged by placing the known terms to the right-hand side of the equation as follows:

$$
\alpha_{i j}+\beta_{i j} \frac{f_{1}^{2}}{f_{j}^{2}}+\gamma_{i j} \frac{f_{1}^{2}}{f_{k}^{2}}=\frac{f_{1}^{2}}{f_{i}^{2}-f_{i} f_{j}}-\frac{f_{1}^{2}}{f_{i} f_{j}-f_{j}^{2}}
$$


The geometry-free condition is achieved by equating the geometry terms in Eqn. 5 to zero, expressed as:

$$
\frac{f_{i}}{f_{i}-f_{j}} \rho-\frac{f_{j}}{f_{i}-f_{j}} \rho+\alpha_{i j} \rho+\beta_{i j} \rho+\gamma_{i j} \rho=0
$$

which results in:

$$
\alpha_{i j}+\beta_{i j}+\gamma_{i j}=\frac{f_{j}-f_{i}}{f_{i}-f_{j}}
$$

The carrier phase coefficients given in Eqn. 4 are known constants due to preservation of the WL ambiguity. Therefore, the measurement noise minimisation is achieved by minimising the sum of the squares of the code coefficients as:

$$
\alpha_{i j}^{2}+\beta_{i j}^{2}+\gamma_{i j}^{2}=M I N
$$

The conditions in Eqns. 7, 9 and 10 are applied to solve for the code coefficients $\alpha_{i j}, \beta_{i j}$ and $\gamma_{i j}$. The resulting geometry and ionosphere-free (GIF) combination contains the integer WL ambiguity presented as:

$$
N_{j i}=N_{i}-N_{j}=\phi_{i}-\phi_{j}+\frac{f_{i}-f_{j}}{c}\left(\alpha_{i j} P_{i}+\beta_{i j} P_{j}+\gamma_{i j} P_{k}\right)+\varepsilon
$$

where the $\varepsilon$ term contains satellite and receiver fractional hardware biases and multipath. The $N_{j i}$ WL ambiguity may now be fixed by averaging multiple epochs of data (Ge et al., 2008; Geng et al., 2010; Laurichesse et al., 2009). The combination coefficients evaluated for different signal combinations for GPS (G), Beidou Phase II (C) and Galileo (E) signals are given in Table 1

The future Beidou Phase III frequencies B1C, B2a and B2b coincide with Galileo E1, E5a and E5b frequencies (Lu and Yao, 2014). Also given is the measurement noise expressed by the std in the proposed and MW combinations as a fraction $\lambda_{W L}$. The std values, in distance units, were computed using $\sigma_{\Phi}=0.002 \mathrm{~m}$ and $\sigma_{\mathrm{P}}=0.4 \mathrm{~m}$ as:

$$
\sigma_{i j}=\sqrt{{\sigma_{\Phi}}^{2}\left(a_{i j}+b_{i j}\right)+{\sigma_{\mathrm{P}}}^{2}\left({\alpha_{i j}}^{2}+{\beta_{i j}}^{2}+\gamma_{i j}{ }^{2}\right)}
$$

Where $a_{i j}=\frac{\mathrm{f}_{\mathrm{i}}{ }^{2}}{\left(\mathrm{f}_{\mathrm{i}}-\mathrm{f}_{j}\right)^{2}}$ and $b_{i j}=\frac{\mathrm{f}_{j}{ }^{2}}{\left(\mathrm{f}_{\mathrm{i}}-\mathrm{f}_{\mathrm{j}}\right)^{2}}$. Figure 1 compares the propagation of measurement noise in this proposed linear combination to the MW combination for GPS with $0.1 m \leq \sigma_{\mathrm{P}} \leq$ $1.5 \mathrm{~m}$ and carrier phase std of $0.002 \mathrm{~m}$ and $0.02 \mathrm{~m}$. These range of std values depict measurement quality during low to high multipath conditions as reported in Liu at el. (2016); Ray et al. (1999) and mapping of the noise from the zenith to low-elevation angles. As shown in Table 1 and Figure 1, the improvements in std when using the proposed combination over MW are $6.7 \%$ for L1-L2, $12.7 \%$ for L1-L5 and $0.7 \%$ for L2-L5. There is substantial improvement in measurement noise propagation for the L1-L5 combination as the pseudorange std increases. At $\sigma_{\mathrm{P}}=1.0 \mathrm{~m}$, the std of the measurement noise in the proposed combination is 0.8 of the wavelength, whereas this std is about the same as the wavelength for MW. The increase in 
carrier phase std has little impact with $\sigma_{\mathrm{P}}>0.2 \mathrm{~m}$ for the WL combinations. The improvement for the L1-L2 proposed combination is also substantial and these results are promising for the rapid estimation of the L1-L2 and L1-L5 ambiguities. The L2-L5 EWL ambiguity, with a measurement noise factor of less than $5 \%$ of the wavelength, is expected to be resolved instantly with both methods.

Figure 2 compares the measurement noise propagation in the proposed linear combination with MW for Beidou Phase II, whereas Figure 3 compares the same for Galileo. Similar improvements in measurement noise propagation for the WL combinations are seen for these constellations.

Table 1: Proposed combination coefficients for GPS, Beidou Phase II and Galileo signals, with the measurement noise propagation compared to MW, and as a fraction of the wide-lane wavelength $\left(\lambda_{W L}\right)$. The std of measurement noise was computed using a carrier phase and pseudorange stds of $0.002 \mathrm{~m}$ and $0.4 \mathrm{~m}$, respectively.

\begin{tabular}{|c|c|c|c|c|c|c|c|c|c|c|}
\hline GNSS & $f_{i}$ & $f_{j}$ & $\alpha$ & $\beta$ & $\gamma$ & $\lambda_{W L}(\mathrm{~m})$ & $\begin{array}{l}\text { Std } \\
\text { proposed } \\
\text { Comb(m) }\end{array}$ & $\begin{array}{l}\text { Std } \\
\text { MW } \\
(\mathrm{m})\end{array}$ & $\begin{array}{l}\text { Std } \\
\text { Improvement } \\
(\%) \text { over MW }\end{array}$ & $\begin{array}{l}\text { Proposed Comb. } \\
\text { Std as fraction } \\
\text { of } \lambda_{W L}\end{array}$ \\
\hline \multirow{3}{*}{$\begin{array}{l}\text { GPS/ } \\
\text { QZSS }\end{array}$} & L2 & L5 & -0.01211 & -0.44499 & -0.5429 & 5.861 & 0.289 & 0.291 & $0.70 \%$ & 0.049 \\
\hline & L1 & L5 & -0.52318 & -0.26734 & -0.20948 & 0.751 & 0.250 & 0.286 & $12.68 \%$ & 0.332 \\
\hline & L1 & $\mathrm{L} 2$ & -0.59833 & -0.24122 & -0.16045 & 0.862 & 0.266 & 0.285 & $6.69 \%$ & 0.309 \\
\hline \multirow{3}{*}{$\begin{array}{l}\text { Beidou } \\
\text { Phase } \\
\text { II }\end{array}$} & B3 & B2 & -0.01995 & -0.55258 & -0.42748 & 4.884 & 0.285 & 0.289 & $1.14 \%$ & 0.058 \\
\hline & B1 & B3 & -0.59733 & -0.14865 & -0.25403 & 1.025 & 0.267 & 0.285 & $6.31 \%$ & 0.260 \\
\hline & $\mathrm{B} 1$ & $\mathrm{~B} 2$ & -0.4972 & -0.21869 & -0.28411 & 0.847 & 0.245 & 0.285 & $13.99 \%$ & 0.290 \\
\hline \multirow{3}{*}{ Galileo } & E5b & E5a & -0.00435 & -0.52751 & -0.46814 & 9.768 & 0.303 & 0.303 & $0.23 \%$ & 0.031 \\
\hline & E1 & $\mathrm{E} 5 \mathrm{~b}$ & -0.58949 & -0.18214 & -0.22837 & 0.814 & 0.263 & 0.286 & $7.75 \%$ & 0.324 \\
\hline & E1 & E5a & -0.54448 & -0.20871 & -0.24681 & 0.751 & 0.253 & 0.286 & $11.37 \%$ & 0.337 \\
\hline
\end{tabular}
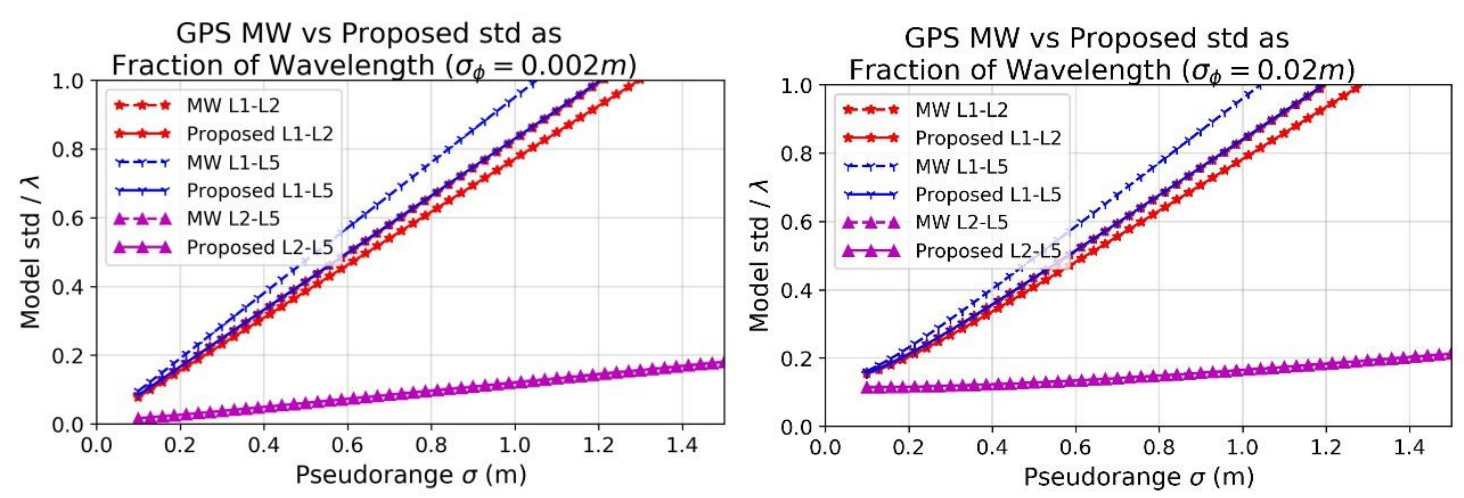

Figure 1: Comparison of the measurement std propagation in the proposed linear combination with the MelbourneWübbena linear combination for GPS with pseudorange std between $0.1 \mathrm{~m}-1.5 \mathrm{~m}$ and carrier phase std of $0.002 \mathrm{~m}$ (left) and 0.02m (right). 

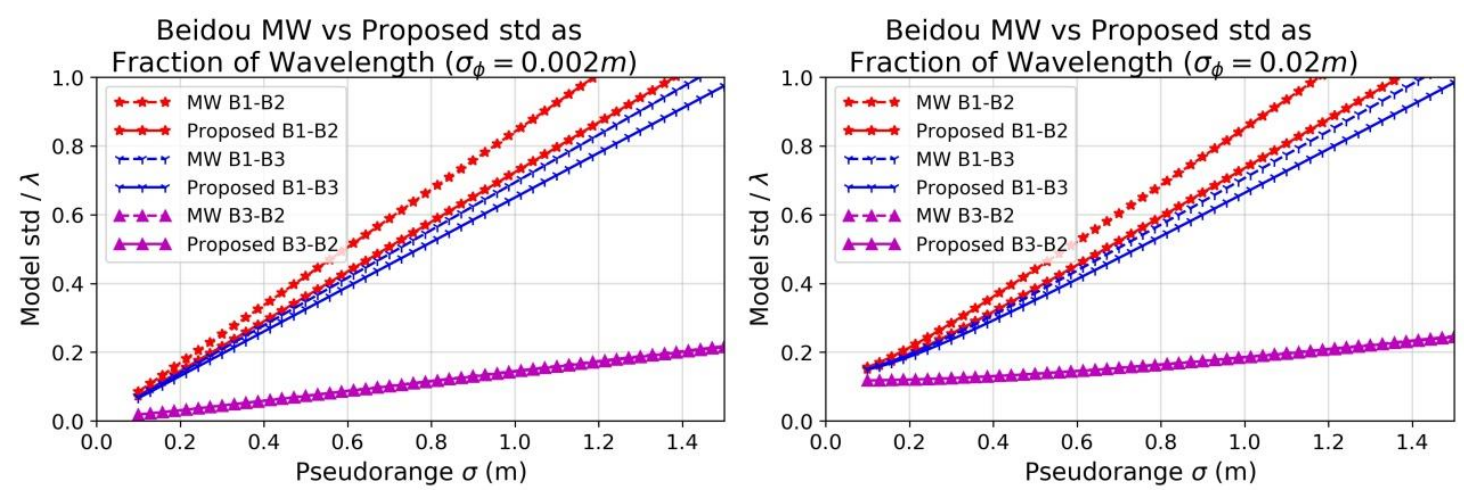

Figure 2: Comparison of the measurement std propagation in the proposed linear combination with the MelbourneWübbena linear combination for Beidou Phase II with pseudorange std between $0.1 \mathrm{~m}-1.5 \mathrm{~m}$ and carrier phase std of $0.002 \mathrm{~m}$ (left) and $0.02 \mathrm{~m}$ (right).

Galileo MW vs Proposed std as

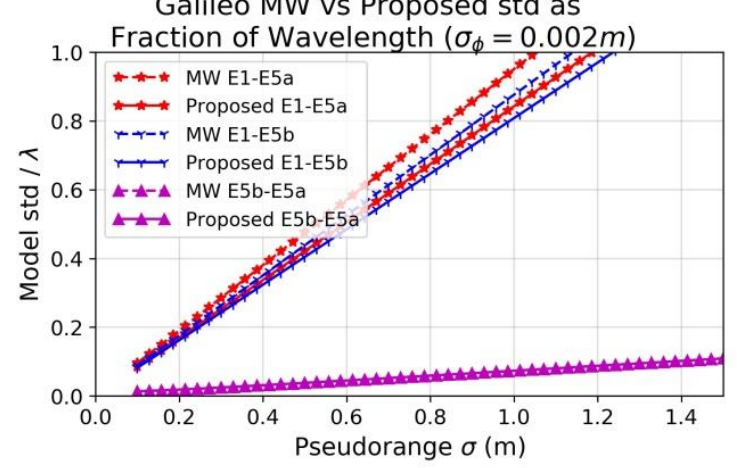

Galileo MW vs Proposed std as

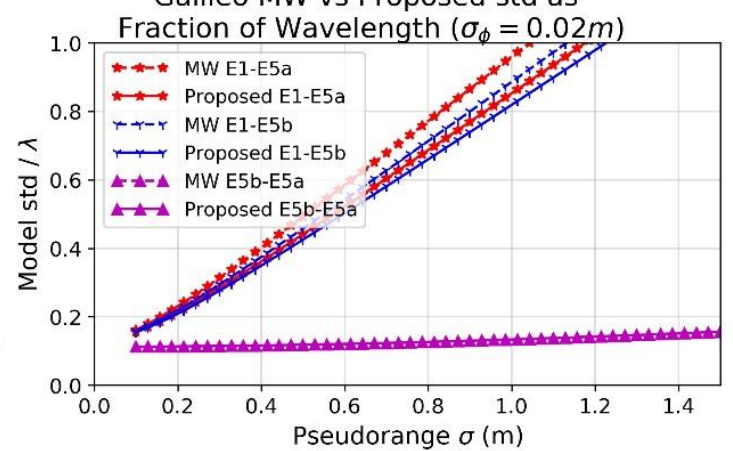

Figure 3: Comparison of the measurement std propagation in the proposed linear combination with the MelbourneWübbena linear combination for Galileo with pseudorange std between $0.1 \mathrm{~m}-1.5 \mathrm{~m}$ and carrier phase std of $0.002 \mathrm{~m}$ (left) and $0.02 \mathrm{~m}$ (right).

\section{PPP with EWL/ WL ambiguities}

Using GPS as an example, this section proposes three PPP models that give decimetre or better accuracy positions instantly after the GPS $\mathrm{N}_{52}$ EWL ambiguity and the $\mathrm{N}_{21}, \mathrm{~N}_{51} \mathrm{WL}$ ambiguities are fixed, which may take less than 2 minutes, using the improved linear combinations proposed in the previous section. The first model uses an IF combination of the three wide-lane phase equations, the second model uses two of the carrier phase wide-lane equations with code measurement on a single frequency, whereas the third model uses one carrier phase wide-lane equation with code measurements on two frequencies. Since the $\mathrm{N}_{52}$ EWL and the $\mathrm{N}_{21}, \mathrm{~N}_{51}$ WL ambiguities are known, the respective wide-lane phase equations may be considered as virtual code measurements with higher precision than the actual codes ( $\mathrm{Li}, 2018)$. The advantage of the proposed models over the float-PPP model is that solution convergence is not required, hence users can get sub-dm level accuracy within just a few minutes of data collection.

\subsection{Ionosphere-Free Combinations of Wide-Lane Carrier Phase only Equations}

In this model, the known $\mathrm{N}_{52}$ EWL and $\mathrm{N}_{21}, \mathrm{~N}_{51}$ WL integer ambiguities are used in their respective carrier phase only wide-lane equations to form an IF linear combination with lowest measurement noise propagation. The carrier phase wide-lane equation, in distance units is expressed as:

$$
\Phi_{W L, j i}=\frac{f_{i}}{f_{i}-f_{j}} \Phi_{i}-\frac{f_{j}}{f_{i}-f_{j}} \Phi_{j}=\rho^{*}+\frac{f_{i}}{f_{j}} I+\frac{c}{f_{i}-f_{j}} N_{j i}+\varepsilon
$$


where the $\rho^{*}$ term includes the geometry, clock errors and the troposphere delay as explained earlier. Given that all the GPS integer EWL and WL ambiguities $N_{j i}$ for $(i, j)$ pairs $(2,5),(1$, $2)$ and $(1,5)$ are known, these are used to derive a corrected wide-lane phase as $\widetilde{\Phi}_{W L, j i}=$ $\Phi_{W L, j i}-\frac{c}{f_{i}-f_{j}} N_{j i}$. This simplifies the three wide-lane phase equations to:

$$
\widetilde{\Phi}_{W L, 52}=\rho^{*}+\frac{f_{2}}{f_{5}} I+\varepsilon ; \widetilde{\Phi}_{W L, 21}=\rho^{*}+\frac{f_{1}}{f_{2}} I+\varepsilon ; \widetilde{\Phi}_{W L, 51}=\rho^{*}+\frac{f_{1}}{f_{5}} I+\varepsilon
$$

A new linear combination is formed from these three equations which is IF, geometry preserving and has lowest measurement noise propagation as:

$$
\widetilde{\Phi}_{I F, 52,21,51}=\alpha_{52} \widetilde{\Phi}_{W L, 52}+\alpha_{21} \widetilde{\Phi}_{W L, 21}+\alpha_{51} \widetilde{\Phi}_{W L, 51}=\rho^{*}+\varepsilon
$$

The first order IF condition is achieved by equating the sum of the ionospheric terms in Eqn. 14 to zero such that:

$$
\alpha_{52} \frac{f_{2}}{f_{5}}+\alpha_{21} \frac{f_{1}}{f_{2}}+\alpha_{51} \frac{f_{1}}{f_{5}}=0
$$

The geometry preserving condition is achieved by:

$$
\alpha_{52}+\alpha_{21}+\alpha_{51}=1
$$

The measurement noise minimisation is achieved by minimising the sum of the squares of the coefficients as:

$$
\alpha_{52}{ }^{2}+\alpha_{21}{ }^{2}+\alpha_{51}{ }^{2}=M I N
$$

A closed-form solution for the coefficient $\alpha_{21}$ is as follows:

$$
\alpha_{21}=\frac{-B}{2 A}
$$

where

$$
\begin{gathered}
A=f_{1}{ }^{2}\left(f_{2}-f_{5}\right)^{2}+\left(f_{2}{ }^{2}-f_{1} f_{5}\right)^{2}+\left(f_{1} f_{2}-f_{2}{ }^{2}\right)^{2} \\
B=-2 f_{1}{ }^{2} f_{2}\left(f_{2}-f_{5}\right)-2{f_{2}}^{2}\left(f_{2}{ }^{2}-f_{1} f_{5}\right)
\end{gathered}
$$

Once $\alpha_{21}$ is known, it may be used to calculate $\alpha_{51}$ as:

$$
\alpha_{51}=\frac{\alpha_{21}\left(f_{2}{ }^{2}-f_{1} f_{5}\right)-f_{2}{ }^{2}}{f_{1} f_{2}-f_{2}{ }^{2}}
$$

and $\alpha_{52}$ may be calculated as: 


$$
\alpha_{52}=\frac{f_{1} f_{2}-\alpha_{21} f_{1}\left(f_{2}-f_{5}\right)}{f_{1} f_{2}-f_{2}{ }^{2}}=1-\alpha_{21}-\alpha_{51}
$$

The coefficients for GPS are evaluated as $\alpha_{52}=4.753204, \alpha_{21}=-1.185810$ and $\alpha_{51}=$ -2.567394 . The measurement noise in this linear combination may be evaluated using error propagation law. Table 2 
Table presents the coefficients for GPS, Beidou Phase II and Galileo signals, along with the measurement noise in the proposed combination using a carrier phase $s t d=0.001 \mathrm{~m}$.

If only the $\mathrm{N}_{52}$ EWL and the $\mathrm{N}_{21}$ WL ambiguities are resolved, the IF formulation may be applied to the two respective carrier phase wide lane equations:

$$
\widetilde{\Phi}_{I F, 52,21}=\alpha_{52} \widetilde{\Phi}_{W L, 52}+\alpha_{21} \widetilde{\Phi}_{W L, 21}=\rho^{*}+\varepsilon
$$

Here, the IF and geometry preserving conditions may be enforced, but not measurement noise minimisation due to use of only two wide-lane equations. After mathematical calculations, the coefficients are evaluated as $\alpha_{52}=5.350453, \alpha_{21}=-4.350453$. Table 2 also shows the measurement noise propagation in this model, which is about $10 \%$ higher than the measurement noise in Eqn. 15. In the unlikely case the EWL ambiguity is not resolved, the $\mathrm{N}_{21}$ and $\mathrm{N}_{51} \mathrm{WL}$ ambiguities must be used to form an IF linear combination $\widetilde{\Phi}_{I F, 21,51}$. The overall measurement noise in this model is about the same as the measurement noise in Eqn. 24, also shown in Table 2. Thus, both these models support instant precise point positioning at the decimetre level when carrier phase std is close to $1 \mathrm{~mm}$, e.g. using high-grade receivers and in low multipath affected environment. As shown in Table 2, the overall measurement noise in Galileo increases at a higher rate compared to GPS and Beidou Phase II. This would make decimetre accurate PPP more challenging with low precision carrier-phase measurements, which occurs in high multipath environments. 
Table 2: Combination coefficients for the ionosphere-free combination with minimum measurement noise propagation of the three wide-lane carrier phase signals. The values are given for GPS, Beidou Phase II and Galileo signals, along with the measurement noise in the combination using a standard deviation of $0.001 \mathrm{~m}$ for carrier phase measurements.

\begin{tabular}{|c|c|c|c|c|c|c|c|c|}
\hline GNSS & $f_{i}$ & $f_{j}$ & $f_{k}$ & Linear combination & $\alpha_{k j}$ & $\alpha_{j i}$ & $\alpha_{k i}$ & $\begin{array}{l}\text { Proposed Comb. Std } \\
\text { (m) }\end{array}$ \\
\hline \multirow{3}{*}{$\begin{array}{l}\text { GPS/ } \\
\text { QZSS }\end{array}$} & \multirow{3}{*}{ L1 } & \multirow{3}{*}{$\mathrm{L} 2$} & \multirow{3}{*}{ L5 } & $\widetilde{\Phi}_{I F, 52,21,51}$ & 4.753204 & -1.185810 & -2.567394 & $0.159 \mathrm{~m}$ \\
\hline & & & & $\widetilde{\Phi}_{I F, 52,21}$ & 5.350453 & -4.350453 & - & $0.180 \mathrm{~m}$ \\
\hline & & & & $\widetilde{\Phi}_{I F, 21,51}$ & - & 24.000000 & -23.000000 & $0.178 \mathrm{~m}$ \\
\hline \multirow{3}{*}{$\begin{array}{l}\text { Beidou } \\
\text { Phase II }\end{array}$} & \multirow{3}{*}{ B1 } & \multirow{3}{*}{ B3 } & \multirow{3}{*}{ B2 } & $\widetilde{\Phi}_{I F, B 2 B 3, B 3 B 1, B 2 B 1}$ & 5.629255 & -1.137165 & -3.492089 & $0.162 \mathrm{~m}$ \\
\hline & & & & $\widetilde{\Phi}_{I F, B 2 B 3, B 3 B 1}$ & 6.844610 & -5.844610 & - & $0.199 \mathrm{~m}$ \\
\hline & & & & $\widetilde{\Phi}_{I F, B 3 B 1, B 2 B 1}$ & - & 20.666667 & -19.666667 & $0.179 \mathrm{~m}$ \\
\hline \multirow{3}{*}{ Galileo } & \multirow{3}{*}{ E1 } & \multirow{3}{*}{$\mathrm{E} 5 \mathrm{~b}$} & \multirow{3}{*}{ E5a } & $\widetilde{\Phi}_{I F, 5 a 5 b, 5 b 1,5 a 1}$ & 4.425670 & -1.359836 & -2.065833 & $0.243 \mathrm{~m}$ \\
\hline & & & & $\widetilde{\Phi}_{I F, 5 a 5 b, 5 b 1}$ & 4.677760 & -3.677760 & - & $0.258 \mathrm{~m}$ \\
\hline & & & & $\widetilde{\Phi}_{I F, 5 b 1,5 a 1}$ & - & 39.333333 & -38.333333 & $0.284 \mathrm{~m}$ \\
\hline
\end{tabular}

\subsection{Ionosphere-Free Combination of Two Wide-Lane Phase Equations and One Code Measurement}

This section proposes a PPP model where two of the carrier phase wide-lane equations are used with a pseudorange measurement on a single frequency to form an IF linear combination with minimum noise propagation. The resolved EWL and WL ambiguities are firstly used to correct the wide-lane phase equations, $\widetilde{\Phi}_{W L, i j}$ and $\widetilde{\Phi}_{W L, k l}$, as shown in the previous section. A new linear combination is formed from these two wide-lane phase equations and a pseudorange measurement on a single frequency. This linear combination is IF, geometry preserving and has lowest measurement noise propagation. It may be expressed as:

$$
\widetilde{\Phi}_{I F, i-m}=\alpha_{i j} \widetilde{\Phi}_{W L, i j}+\alpha_{k l} \widetilde{\Phi}_{W L, k l}+\alpha_{m} \widetilde{P}_{m}=\rho^{*}+\varepsilon
$$

where $\tilde{P}_{m}$ is a BSSD pseudorange measurement on frequency $m$. When mixing the pseudorange measurement with the carrier phase wide-lane equations, it must be corrected for the satellite clock error and differential code bias (DCB), which are available, for instance, as part of the IGS Multi-GNSS Experiment (MGEX) products at the web-site ftp://cddis.nasa.gov/gnss/products/bias/. The first order IF condition is achieved by equating the sum of the ionospheric terms in Eqn. 25 to zero such that

$$
\alpha_{i j} \frac{f_{i}}{f_{j}}+\alpha_{k l} \frac{f_{k}}{f_{l}}+\alpha_{m} \frac{f_{1}^{2}}{f_{m}{ }^{2}}=0
$$

The geometry preserving condition is achieved by:

$$
\alpha_{i j}+\alpha_{k l}+\alpha_{m}=1
$$

Assuming that the code noise is higher than the phase noise by a constant factor, $n$, i.e. $\sigma_{P}=$ $n \sigma_{\phi}$, the noise minimisation in the combination is achieved by minimising the amplification factors with the following condition:

$$
\alpha_{i j}^{2}+\alpha_{j k}^{2}+n^{2} \alpha_{m}^{2}=\mathrm{MIN}
$$


where the code and carrier-phase measurements to be weighted according to their measurement noise. The closed-form analytical solution for the coefficient $\alpha_{m}$ is given as:

$$
\alpha_{m}=\frac{-B}{2 A}
$$

where

with

$$
\begin{gathered}
A=X_{1}{ }^{2}+X_{2}{ }^{2}+X_{3}{ }^{2} \\
B=2 X_{1} f_{m}{ }^{2} f_{k} f_{j}-2 X_{2} f_{m}{ }^{2} f_{i} f_{l}
\end{gathered}
$$

$$
\begin{gathered}
X_{1}=f_{1}{ }^{2} f_{j} f_{k}-f_{m}{ }^{2} f_{k} f_{j} \\
X_{2}=f_{m}{ }^{2} f_{i} f_{l}-f_{1}{ }^{2} f_{j} f_{l} \\
X_{3}=f_{m}{ }^{2}\left(f_{k} f_{j}-f_{i} f_{l}\right)
\end{gathered}
$$

Once $\alpha_{m}$ is known, it is used to calculate $\alpha_{k l}$ as:

$$
\alpha_{k l}=\frac{\alpha_{m} X_{2}-f_{m}{ }^{2} f_{i} f_{l}}{X_{3}}
$$

and $\alpha_{i j}$ is calculated as:

$$
\alpha_{i j}=\frac{\alpha_{m} X_{1}-f_{m}{ }^{2} f_{k} f_{j}}{X_{3}}=1-\alpha_{k l}-\alpha_{m}
$$

The overall measurement noise in Eqn. 25 is evaluated using error propagation law as:

$$
\sigma_{\widetilde{\Phi}_{I F, i-m}}=\sqrt{\sigma_{\phi}{ }^{2}\left\{\left(\frac{\alpha_{i j} f_{i}}{f_{i}-f_{j}}\right)^{2}+\left(\frac{\alpha_{i j} f_{j}}{f_{i}-f_{j}}\right)^{2}+\left(\frac{\alpha_{k l} f_{k}}{f_{k}-f_{l}}\right)^{2}+\left(\frac{\alpha_{k l} f_{l}}{f_{k}-f_{l}}\right)^{2}\right\}+\sigma_{P}{ }^{2} \alpha_{m}{ }^{2}}
$$

Table 3 shows the combination coefficients for the proposed linear combination for GPS and QZSS, Beidou Phase II and Galileo signals. The overall measurement noise is also given in the last column using a carrier phase std $\left(\sigma_{\phi}\right)=0.001 \mathrm{~m}$ and pseudorange std $\left(\sigma_{P}\right)=0.400 \mathrm{~m}$; hence $n=100$. As the table shows, the lowest overall measurement noise is achieved with $\widetilde{\Phi}_{W L, 15}$ and $\widetilde{\Phi}_{W L, 25}$ for GPS, with $\widetilde{\Phi}_{W L, B 1 B 2}$ and $\widetilde{\Phi}_{W L, B 3 B 2}$ for Beidou Phase II, and with $\widetilde{\Phi}_{W L, E 1 E 5 a}$ and $\widetilde{\Phi}_{W L, E 5 a E 5 b}$ for Galileo. For GPS and Beidou Phase II, the overall measurement noise is $0.15 \mathrm{~m}$ at best, whereas for Galileo it is $0.20 \mathrm{~m}$. These values have improved slightly compared to the overall measurement noise in the carrier phase only linear combination developed in the previous section. As shown in the last column of Table 3, selection of the pseudorange measurement frequency, $f_{m}$, has a negligible impact on the overall noise in the proposed combination.

Table 3: Combination coefficients for the ionosphere-free combination with minimum measurement noise propagation of the two wide-lane carrier-phase and single-code signal. The values are given for GPS, Beidou Phase II and Galileo signals, along with the overall measurement noise with a standard deviation of $0.001 \mathrm{~m}$ for carrier phase and $0.400 \mathrm{~m}$ for code measurements. 


\begin{tabular}{|c|c|c|c|c|c|c|c|c|c|}
\hline GNSS & $f_{i}$ & $f_{j}$ & $f_{k}$ & $f_{l}$ & $f_{m}$ & $\alpha_{i j}$ & $\alpha_{k l}$ & $\alpha_{m}$ & $\begin{array}{l}\text { Proposed Comb. Std } \\
\text { (m) }\end{array}$ \\
\hline \multirow{9}{*}{$\begin{array}{l}\text { GPS/ } \\
\text { QZSS }\end{array}$} & L1 & $\mathrm{L} 2$ & $\mathrm{~L} 1$ & L5 & $\mathrm{L} 1$ & 23.990026 & -22.991667 & 0.001641 & 0.1784 \\
\hline & L1 & $\mathrm{L} 2$ & L1 & L5 & $\mathrm{L} 2$ & 23.990272 & -22.988508 & -0.001763 & 0.1784 \\
\hline & $\mathrm{L} 1$ & $\mathrm{~L} 2$ & $\mathrm{~L} 1$ & L5 & L5 & 23.979390 & -22.976857 & -0.002532 & 0.1783 \\
\hline & L1 & $\mathrm{L} 2$ & $\mathrm{~L} 2$ & L5 & L1 & -4.350445 & 5.350401 & 0.000044 & 0.1796 \\
\hline & L1 & $\mathrm{L} 2$ & $\mathrm{~L} 2$ & L5 & $\mathrm{L} 2$ & -4.350154 & 5.350273 & -0.000119 & 0.1796 \\
\hline & L1 & $\mathrm{L} 2$ & $\mathrm{~L} 2$ & L5 & L5 & -4.349965 & 5.350121 & -0.000156 & 0.1796 \\
\hline & $L 1$ & $L 5$ & $L 2$ & $L 5$ & $L 1$ & -3.529407 & 4.529371 & 0.000036 & 0.1516 \\
\hline & $L 1$ & L5 & $L 2$ & L5 & $L 2$ & -3.52926 & 4.529334 & -0.000074 & 0.1516 \\
\hline & $L 1$ & $L 5$ & $L 2$ & $L 5$ & $L 5$ & -3.52916 & 4.529259 & -0.000099 & 0.1516 \\
\hline \multirow{9}{*}{$\begin{array}{l}\text { Beidou } \\
\text { Phase II }\end{array}$} & B1 & B3 & B1 & B2 & B1 & 20.661709 & -19.662767 & 0.001058 & 0.1794 \\
\hline & B1 & B3 & $\mathrm{B} 1$ & $\mathrm{~B} 2$ & B3 & 20.663081 & -19.662067 & -0.001014 & 0.1794 \\
\hline & B1 & B3 & B1 & $\mathrm{B} 2$ & $\mathrm{~B} 2$ & 20.65667 & -19.655021 & -0.00165 & 0.1794 \\
\hline & B1 & B3 & B3 & $\mathrm{B} 2$ & B1 & -5.844592 & 6.844526 & 0.000065 & 0.1994 \\
\hline & $\mathrm{B} 1$ & B3 & B3 & $\mathrm{B} 2$ & B3 & -5.844193 & 6.844355 & -0.000162 & 0.1994 \\
\hline & B1 & B3 & B3 & $\mathrm{B} 2$ & $\mathrm{~B} 2$ & -5.84381 & 6.844042 & -0.000231 & 0.1993 \\
\hline & $B 1$ & $B 2$ & B3 & $B 2$ & $B 1$ & -4.335655 & 5.335609 & 0.000046 & 0.1541 \\
\hline & $B 1$ & $B 2$ & B3 & $B 2$ & B3 & -4.335507 & 5.335589 & -0.000082 & 0.1541 \\
\hline & $B 1$ & $B 2$ & B3 & $B 2$ & $B 2$ & -4.335352 & 5.335474 & -0.000122 & 0.1541 \\
\hline \multirow{9}{*}{ Galileo } & E1 & E5a & E1 & $\mathrm{E} 5 \mathrm{~b}$ & E1 & -38.2922 & 39.287607 & 0.004591 & 0.2836 \\
\hline & E1 & E5a & E1 & $\mathrm{E} 5 \mathrm{~b}$ & E5a & -38.23728 & 39.24398 & -0.006699 & 0.2833 \\
\hline & E1 & E5a & E1 & $\mathrm{E} 5 \mathrm{~b}$ & $\mathrm{E} 5 \mathrm{~b}$ & -38.26992 & 39.275339 & -0.005423 & 0.2835 \\
\hline & E1 & $E 5 a$ & $E 5 a$ & $E 5 b$ & E1 & -2.673339 & 3.673319 & 0.000020 & 0.2022 \\
\hline & E1 & $E 5 a$ & $E 5 a$ & $E 5 b$ & $E 5 a$ & -2.673189 & 3.673255 & -0.000066 & 0.2022 \\
\hline & E1 & $E 5 a$ & $E 5 a$ & $E 5 b$ & $E 5 b$ & -2.673225 & 3.673281 & -0.000056 & 0.2022 \\
\hline & E1 & $\mathrm{E} 5 \mathrm{~b}$ & E5a & $\mathrm{E} 5 \mathrm{~b}$ & E1 & -2.94872 & 3.948698 & 0.000021 & 0.2175 \\
\hline & E1 & $\mathrm{E} 5 \mathrm{~b}$ & E5a & $\mathrm{E} 5 \mathrm{~b}$ & E5a & -2.948515 & 3.948597 & -0.000082 & 0.2175 \\
\hline & E1 & $\mathrm{E} 5 \mathrm{~b}$ & $\mathrm{E} 5 \mathrm{a}$ & $\mathrm{E} 5 \mathrm{~b}$ & $\mathrm{E} 5 \mathrm{~b}$ & -2.948563 & 3.948633 & -0.00007 & 0.2175 \\
\hline
\end{tabular}

\subsection{Ionosphere-Free Combination of One Wide-Lane Phase Equation and Two Code Measurements}

This section proposes a third PPP model where one of the carrier phase wide-lane equation is used with pseudorange measurements on two frequencies to form an IF linear combination with a minimum noise propagation. This linear combination is IF, geometry preserving and has lowest measurement noise propagation and is expressed as:

$$
\widetilde{\Phi}_{I F, i-l}=\alpha_{i j} \widetilde{\Phi}_{W L, i j}+\alpha_{k} \tilde{P}_{k}+\alpha_{l} \widetilde{P}_{l}=\rho^{*}+\varepsilon
$$

Where $\widetilde{P}_{k}$ and $\widetilde{P}_{l}$ are BSSD pseudorange measurements on frequencies $k$ and $l$, corrected for satellite clock and hardware delay errors obtained from external sources. The first order IF condition is achieved by equating the sum of the ionospheric terms in Eqn. 38 to zero such that

$$
\alpha_{i j} \frac{f_{i}}{f_{j}}+\alpha_{k} \frac{f_{1}^{2}}{f_{k}{ }^{2}}+\alpha_{l} \frac{f_{1}^{2}}{f_{l}^{2}}=0
$$


The geometry preserving condition is achieved by:

$$
\alpha_{i j}+\alpha_{k}+\alpha_{l}=1
$$

The noise minimisation in the combination is achieved by minimising the amplification factors with the following condition:

$$
\alpha_{i j}^{2}+n^{2} \alpha_{k}^{2}+n^{2} \alpha_{l}^{2}=\operatorname{MIN}
$$

The closed-form analytical solution for the coefficient $\alpha_{l}$ is given as:

$$
\alpha_{l}=\frac{-B}{2 A}
$$

where

with

$$
\begin{gathered}
A=\left(-X_{1}-X_{2}\right)^{2}+n^{2} X_{1}{ }^{2} \\
B=-2\left(-X_{2}-X_{1}\right)\left(X_{2}+X_{3}\right)-2 n^{2} X_{1} X_{3}
\end{gathered}
$$

$$
\begin{gathered}
X_{1}=f_{1}{ }^{2} f_{k}{ }^{2}\left(f_{i}-f_{j}\right) \\
X_{2}=f_{1}{ }^{2}\left(f_{j} f_{1}{ }^{2}-f_{i} f_{k}{ }^{2}\right) \\
X_{3}=f_{1}{ }^{2} f_{k}{ }^{2} f_{i}
\end{gathered}
$$

Once $\alpha_{l}$ is known, it is used to calculate $\alpha_{i j}$ as:

$$
\alpha_{i j}=\frac{\alpha_{l}\left(-X_{2}-X_{1}\right)+\left(X_{2}+X_{3}\right)}{X_{2}}
$$

and $\alpha_{k}$ is calculated as:

$$
\alpha_{k}=\frac{\alpha_{l} X_{1}-X_{3}}{X_{2}}=1-\alpha_{i j}-\alpha_{l}
$$

The overall measurement noise in Eqn. 38 is evaluated as:

$$
\sigma_{\widetilde{\Phi}_{I F, i-l}}=\sqrt{\sigma_{\phi}{ }^{2}\left\{\left(\frac{\alpha_{i j} f_{i}}{f_{i}-f_{j}}\right)^{2}+\left(\frac{\alpha_{i j} f_{j}}{f_{i}-f_{j}}\right)^{2}\right\}+{\sigma_{P}}^{2} \alpha_{k}{ }^{2}+{\sigma_{P}}^{2} \alpha_{l}{ }^{2}}
$$

Table 4 shows the combination coefficients for the proposed linear combination for GPS and QZSS, Beidou Phase II and Galileo signals. The overall measurement noise is given in the last column using a carrier phase std $\left(\sigma_{\phi}\right)=0.001 \mathrm{~m}$ and pseudorange std $\left(\sigma_{P}\right)=0.300 \mathrm{~m}$; hence $n=300$. As the table shows, the lowest overall measurement noise is achieved with $\widetilde{\Phi}_{W L, 25}$ and $(k, l)=L 1, L 5$ or $L 1, L 5$ for GPS, with $\widetilde{\Phi}_{W L, B 3 B 2}$ and $(k, l)=B 1, B 2$ or $B 3, B 2$ for Beidou Phase II, and with $\widetilde{\Phi}_{W L, E 5 a E 5 b}$ and $(k, l)=E 1, E 5 a$ or $E 5 b, E 5 a$ for Galileo. For GPS, the overall measurement noise is $0.425 \mathrm{~m}$ at best, for Beidou Phase II the best overall noise is $0.513 \mathrm{~m}$, and for Galileo the best noise is $0.377 \mathrm{~m}$. These values are significantly worse than the 
previous two proposed combinations. However, further analysis in the next section will show that this method surpasses the performance of the previous methods as well as the method of Li et al. (2014) where the carrier phase std increases above $0.005 \mathrm{~m}$.

Table 4: Combination coefficients for the ionosphere-free combination with minimum measurement noise propagation of the single wide-lane carrier phase and code signals on two frequencies. The values are given for GPS, Beidou Phase II and Galileo signals, along with the overall measurement noise with a standard deviation of $0.001 \mathrm{~m}$ for carrier phase and $0.300 \mathrm{~m}$ for code measurements. The results given for overall measurement noise less than $1.0 \mathrm{~m}$.

\begin{tabular}{|c|c|c|c|c|c|c|c|c|}
\hline GNSS & $f_{i}$ & $f_{j}$ & $f_{k}$ & $f_{l}$ & $\alpha_{i j}$ & $\alpha_{k}$ & $\alpha_{l}$ & $\begin{array}{l}\text { Proposed Comb. Std } \\
\text { (m) }\end{array}$ \\
\hline \multirow{8}{*}{$\begin{array}{l}\text { GPS/ } \\
\text { QZSS }\end{array}$} & L1 & L2 & L1 & L5 & 3.516366 & 0.000183 & -2.516549 & 0.7552 \\
\hline & L1 & L2 & L2 & L5 & 3.516656 & -0.000018 & -2.516638 & 0.7553 \\
\hline & L1 & L5 & L1 & L5 & 3.948384 & 0.000191 & -2.948575 & 0.8848 \\
\hline & L1 & L5 & L2 & L5 & 3.948726 & -0.000024 & -2.948702 & 0.8848 \\
\hline & L2 & L5 & L1 & L2 & 2.728519 & 0.000580 & -1.729099 & 0.5266 \\
\hline & $L 2$ & $L 5$ & L1 & $L 5$ & 2.391088 & 0.000569 & -1.391657 & 0.4250 \\
\hline & L2 & L5 & L2 & L5 & 2.391691 & -0.000007 & -1.391685 & 0.4250 \\
\hline & L2 & L5 & L5 & L2 & 2.729143 & 0.000006 & -1.729148 & 0.5266 \\
\hline \multirow{6}{*}{$\begin{array}{l}\text { Beidou } \\
\text { Phase II }\end{array}$} & B1 & B3 & B1 & B2 & 3.785371 & 0.000211 & -2.785581 & 0.8361 \\
\hline & $\mathrm{B} 1$ & B3 & B3 & B2 & 3.785701 & -0.000027 & -2.785674 & 0.8361 \\
\hline & B3 & $B 2$ & $B 1$ & $B 2$ & 2.690084 & 0.000505 & -1.690589 & 0.5130 \\
\hline & B3 & B2 & B1 & B3 & 3.265933 & 0.000525 & -2.266458 & 0.6863 \\
\hline & $B 3$ & $B 2$ & $B 3$ & $B 2$ & 2.690633 & -0.000011 & -1.690622 & 0.5130 \\
\hline & B3 & B2 & B2 & B3 & 3.266518 & 0.000008 & -2.266526 & 0.6863 \\
\hline \multirow{10}{*}{ Galileo } & E1 & E5a & E1 & E5a & 3.948384 & 0.000191 & -2.948575 & 0.8848 \\
\hline & E1 & E5a & E5b & E5a & 3.948720 & -0.000012 & -2.948708 & 0.8848 \\
\hline & E1 & E5b & E1 & E5a & 3.673037 & 0.000185 & -2.673222 & 0.8022 \\
\hline & E1 & E5b & E1 & $\mathrm{E} 5 \mathrm{~b}$ & 4.277402 & 0.000213 & -3.277615 & 0.9836 \\
\hline & E1 & E5b & E5a & $\mathrm{E} 5 \mathrm{~b}$ & 4.277780 & 0.000008 & -3.277787 & 0.9836 \\
\hline & E1 & E5b & E5b & E5a & 3.673339 & -0.000010 & -2.673329 & 0.8022 \\
\hline & $E 5 a$ & $E 5 b$ & E1 & $E 5 a$ & 2.191201 & -0.000823 & -1.190378 & 0.3768 \\
\hline & E5a & E5b & E1 & E5b & 2.338263 & -0.000819 & -1.337444 & 0.4213 \\
\hline & E5a & E5b & E5a & $\mathrm{E} 5 \mathrm{~b}$ & 2.337473 & 0.000003 & -1.337476 & 0.4213 \\
\hline & $E 5 a$ & $E 5 b$ & $E 5 b$ & $E 5 a$ & 2.190404 & -0.000003 & -1.190401 & 0.3768 \\
\hline
\end{tabular}

\section{Analysis of The Results}

This section presents analysis of the proposed method for estimating the EWL and WL integer ambiguities and the PPP models that use these ambiguities.

\subsection{Analysis of the EWL and WL Integer Ambiguity Estimation}

The proposed method for estimating EWL and WL integer ambiguities is analysed using simulated test data at four Australian sites, namely Hobart (HOB2), Alice Springs (ALIC), Yarragadee (YAR2) and Townsville (TOW2). Although GPS data is used as an example, these procedures can be applied for other GNSS constellations that have triple-frequency signals such as Beidou, Galileo and QZSS. The data used was for a 24-hour period, at an epoch rate of 15 seconds, with carrier phase std of $0.002 \mathrm{~m}$ and pseudorange std of $0.4 \mathrm{~m}$. The BSSD 
measurements were used in this analysis, where the measurement noise after the differencing increases by a factor of $\sqrt{2}$.

Firstly, the performance of the improved linear combinations proposed in Section 3 is compared with the standard MW approach for estimating the EWL and WL ambiguities. Figure 4 compares the time series and histogram for the $N_{51} \mathrm{WL}$ ambiguities at YAR2 for all observed satellite pairs, where the mean value is subtracted to isolate the measurement noise. The left plots apply to the MW method whereas the right plots are for the proposed model. As shown, the std of the estimated $N_{51}$ WL ambiguity in the proposed method improved by $6.7 \%$. Figure 5 shows the same results for the $N_{51}$ WL ambiguity, which improved by $13.3 \%$. Figure 6 illustrates the results for the $N_{52}$ EWL ambiguity, which showed marginal improvement, as expected from Table 1. Similar improvements were also found at the other sites.
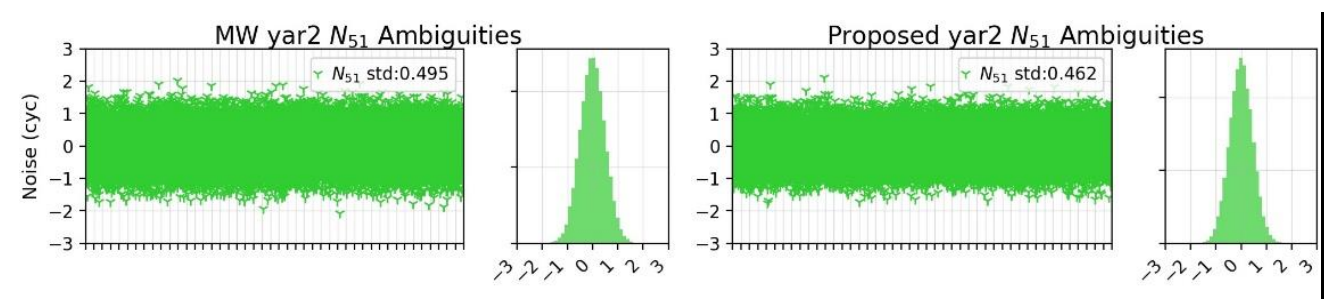

Figure 4: Time series and histogram of the single differenced $N_{51}$ WL ambiguities with the MW method (left) and the proposed method (right) at site YAR2 for all observed satellite pairs.
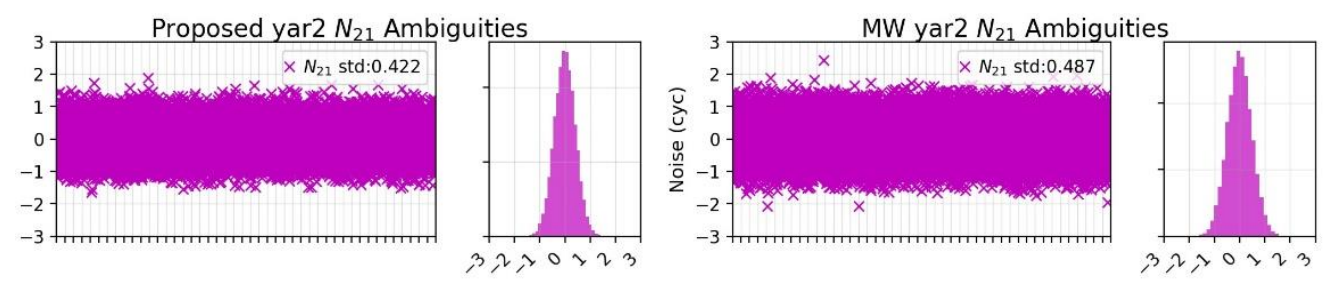

Figure 5: Time series and histogram of the single differenced $N_{21}$ WL ambiguities with the MW method (left) and the proposed method (right) at site YAR2 for all observed satellite pairs.
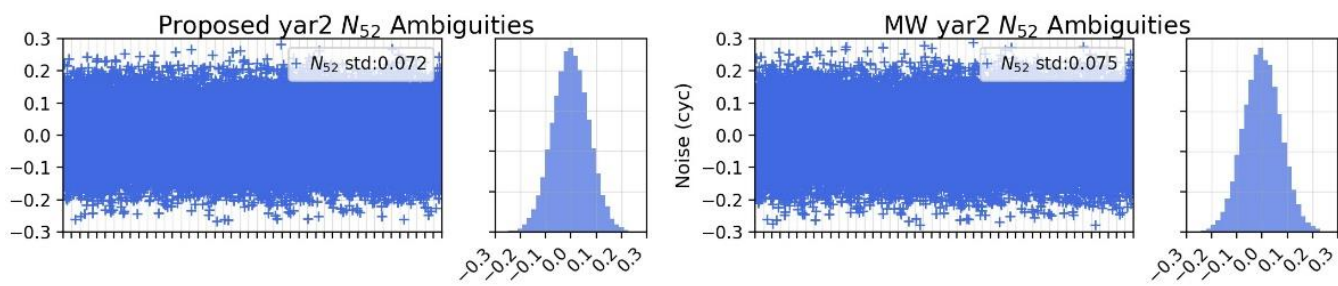

Figure 6: Time series and histogram of the single differenced $N_{52}$ WL ambiguities with the MW method (left) and the proposed method (right) at site YAR2 for all observed satellite pairs.

Secondly, analysis of the time taken to fix the EWL and WL ambiguities was undertaken. This is defined as the elapsed time for the average integer rounded ambiguities to attain a constant value equal to the overall mean for the satellite pair. Figure 7 compares histograms of the number of epochs required to fix the EWL and WL ambiguities for the proposed method and the MW method for all the satellite pairs that were observed at the four sites during the 24-hour observation. As shown, the $\mathrm{N}_{52}$ EWL ambiguities were fixed to the correct integers within a single epoch for both methods. When using the proposed method, the $\mathrm{N}_{21}$ WL ambiguities were fixed correctly within a single epoch for $88.9 \%$ of cases, compared to $83.5 \%$ of cases for the MW method. The $\mathrm{N}_{51}$ WL ambiguities were fixed correctly within a single epoch for $86.0 \%$ of cases with the proposed approach, compared to $80.1 \%$ of cases for the MW method. Table 
4 shows the cumulative percentage of the EWL and WL ambiguities that were fixed with respect to the number of epochs of data collected for MW and proposed methods, respectively. As shown, the performance of the proposed method is superior to MW, particularly for the $\mathrm{N}_{51}$ WL ambiguity, followed by the $\mathrm{N}_{21}$ WL ambiguity. For the proposed method, all the EWL and WL ambiguities were fixed with just seven epochs of data, which amounts to less than 2 minutes. Hence, the proposed PPP method may be applied within just two minutes of data collection that is required to fix the EWL and WL ambiguities.

Table 4: Cumulative percentage of the EWL/ WL ambiguities fixed with respect to the number of epochs of data, for MW and proposed methods.

\begin{tabular}{|c|l|l|l|l|l|l|l|l|}
\hline \multirow{2}{*}{$\begin{array}{l}\text { EWL/ WL } \\
\text { Ambiguity }\end{array}$} & \multirow{2}{*}{ Method } & \multicolumn{6}{|l|}{ Cumulative percentage of EWL/ WL ambiguities fixed wrt number of epochs } \\
\cline { 2 - 9 } & 1 & 2 & 3 & 4 & 5 & 6 \\
\hline $\mathrm{N}_{51}$ & MW & $80.1 \%$ & $89.2 \%$ & $93.9 \%$ & $96.6 \%$ & $97.6 \%$ & $98.6 \%$ \\
\hline & Proposed & $86.0 \%$ & $94.2 \%$ & $97.4 \%$ & $98.9 \%$ & $99.1 \%$ & $99.8 \%$ & $100.0 \%$ \\
\hline $\mathrm{N}_{21}$ & MW & $83.5 \%$ & $93.1 \%$ & $96.8 \%$ & $98.3 \%$ & $99.8 \%$ & $100.0 \%$ & - \\
\hline & Proposed & $88.9 \%$ & $95.3 \%$ & $97.8 \%$ & $99.3 \%$ & $99.3 \%$ & $100.0 \%$ & - \\
\hline $\mathrm{N}_{52}$ & MW & $100.0 \%$ & - & - & - & - & - & - \\
\hline & Proposed & $100.0 \%$ & - & - & - & - & - & - \\
\hline
\end{tabular}
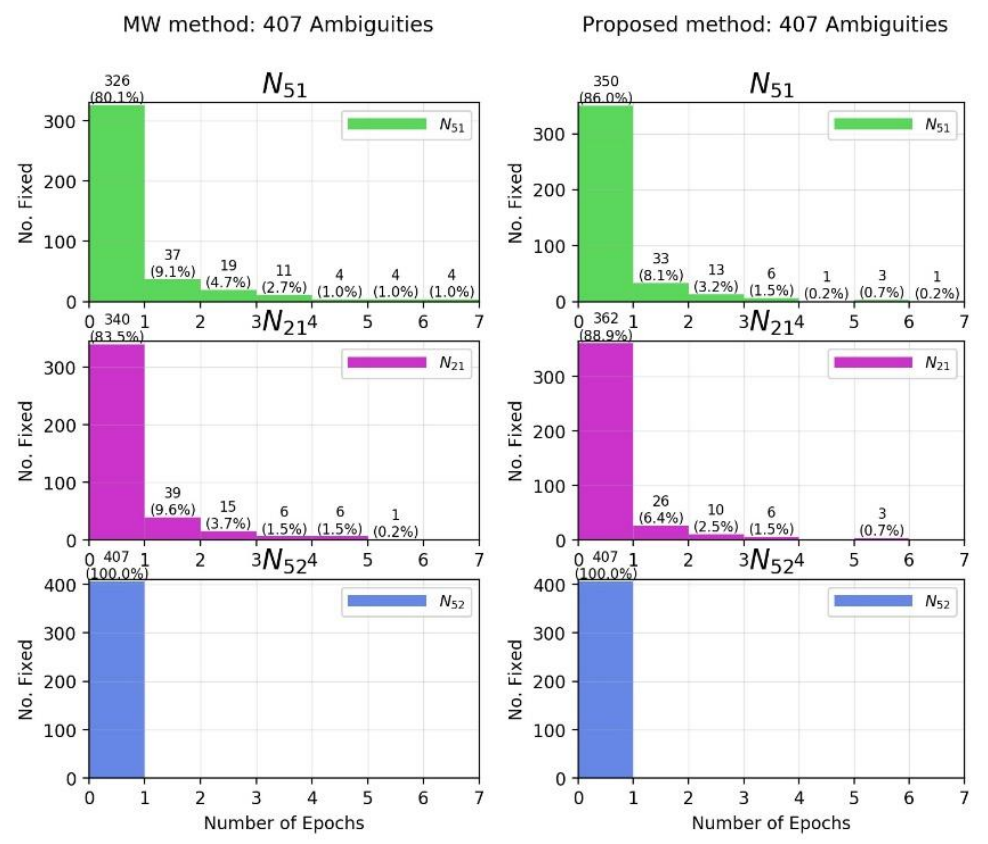

Figure 7: Histogram of the number of cases where the wide-lane (top, middle) and extra wide-lane (bottom) ambiguities were correctly fixed using the MW method (left) and proposed method (right). The x-axis shows the number of epochs required to fix ambiguities and the $y$ axis shows the number of instances (percentage in brackets) of correct ambiguity fixing.

\subsection{Analysis of convergence time for fixing the $W L$ ambiguities}

This section analyses the probability of WL ambiguity resolution success rate (Ps) with a single epoch, followed by analysis of the number of uncorrelated epochs required to fix these ambiguities. As shown in the previous section, resolving the EWL is not an issue since it is normally fixed in a single epoch. The WL ambiguities are directly estimated by rounding the sequential average of the proposed linear combination over several epochs in the measurement domain. This approach is less optimal than the Integer Least Squares (ILS) method, but its advantage is that the ambiguity estimation does not require a search, but is computed directly (Teunissen, 1998). Since the noise in the proposed linear combinations is significantly lower as a fraction of the effective wavelength (35\% of the wavelength at worst - see Table 1), the WL ambiguities may be fixed through rounding sequential averages over a few epochs. The 
lower bound of the Ps with the proposed model, as well as the MW model can be explicitly evaluated by the equation (Teunissen, 1998):

$$
P_{I R(\widetilde{N}=N)}=2 \Phi\left(\frac{1}{2 \sigma_{\widehat{N}}}\right)-1.0
$$

where $\breve{N}, \widehat{N}$ and $\mathrm{N}$ are the integer rounded, float and the correct WL integer ambiguity and $\Phi$ is the cumulative normal distribution function. The Ps rate with a single epoch is assessed for the GPS $N_{21}$ and $N_{51}$ WL ambiguities for the proposed model and the MW model, respectively. This was done for $\sigma_{\Phi}=0.002 \mathrm{~m}$ and $\sigma_{\Phi}=0.02 \mathrm{~m}$, and $\sigma_{\mathrm{P}}$ in the range 0.1 to $1.5 \mathrm{~m}$. Results for the $N_{21}$ WL success rate, shown in Figure 8, indicate that the proposed model gives an improvement over the MW model by approximately $3 \%$, especially when $\sigma_{\mathrm{P}}$ increases above $0.4 \mathrm{~m}$. This applies for both $\sigma_{\Phi}=0.002 \mathrm{~m}$ and $\sigma_{\Phi}=0.02 \mathrm{~m}$, the difference between the two stds being not substantial. Realistically, a Ps of $99.9 \%$ is required when fixing the WL ambiguity with a single epoch. When using the proposed model, the $\sigma_{\mathrm{P}}$ must be less than $0.18 \mathrm{~m}$ to achieve this, with $\sigma_{\Phi}=0.002 \mathrm{~m}$. This is possible with the modernised signals of the GNSS systems and advancements in receiver tracking technology. When $\sigma_{\Phi}=0.02 \mathrm{~m}$, as shown in Figure 8 (right), a Ps of $99.9 \%$ can be achieved with the proposed model if $\sigma_{\mathrm{P}} \leq 0.1 \mathrm{~m}$. Thus, reducing the pseudorange measurement noise is the key to resolving the WL ambiguities with a single epoch. The $N_{51}$ WL success rate results, as shown in Figure 9, shows that the proposed model gives a more substantial improvement of $6 \%$ over the MW model. To achieve a Ps of $99.9 \%$ with a single epoch also requires $\sigma_{\mathrm{P}} \leq 0.18 \mathrm{~m}$, with $\sigma_{\Phi}=0.002 \mathrm{~m}$. When $\sigma_{\Phi}=0.02 \mathrm{~m}$, the Ps of $99.9 \%$ was not achieved with a single epoch with $\sigma_{\mathrm{P}}=0.1 \mathrm{~m}$.
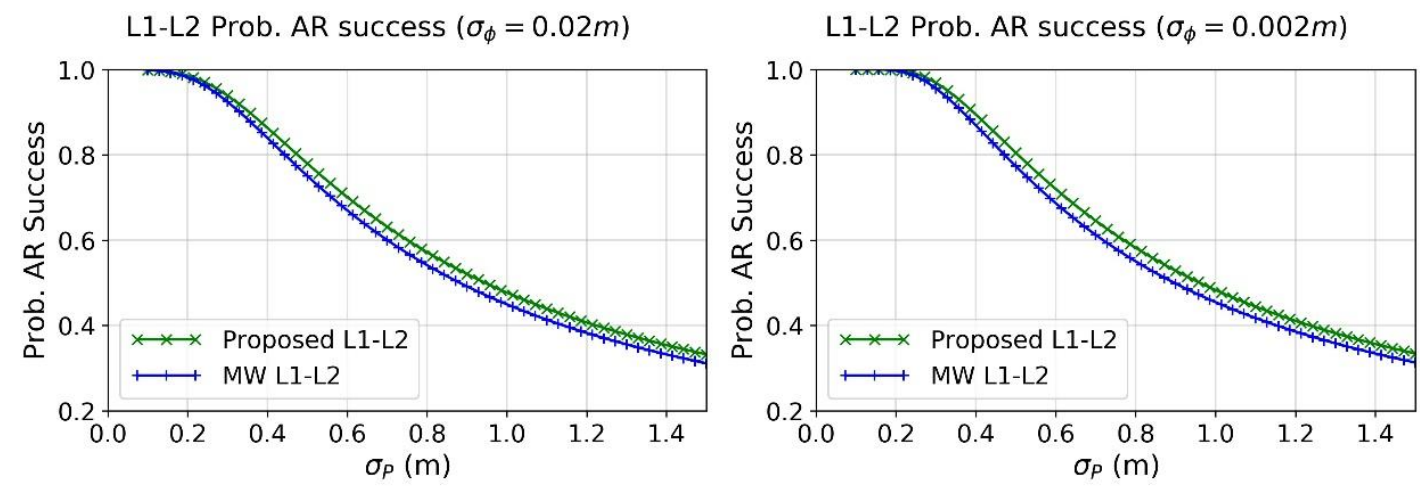

Figure 8: Probability of GPS $N_{21}$ WL integer ambiguity resolution success rate with single epoch data for the proposed model and the MW model with $\sigma_{\Phi}=0.002 m$ (left) and $\sigma_{\Phi}=0.02 m$ (right).
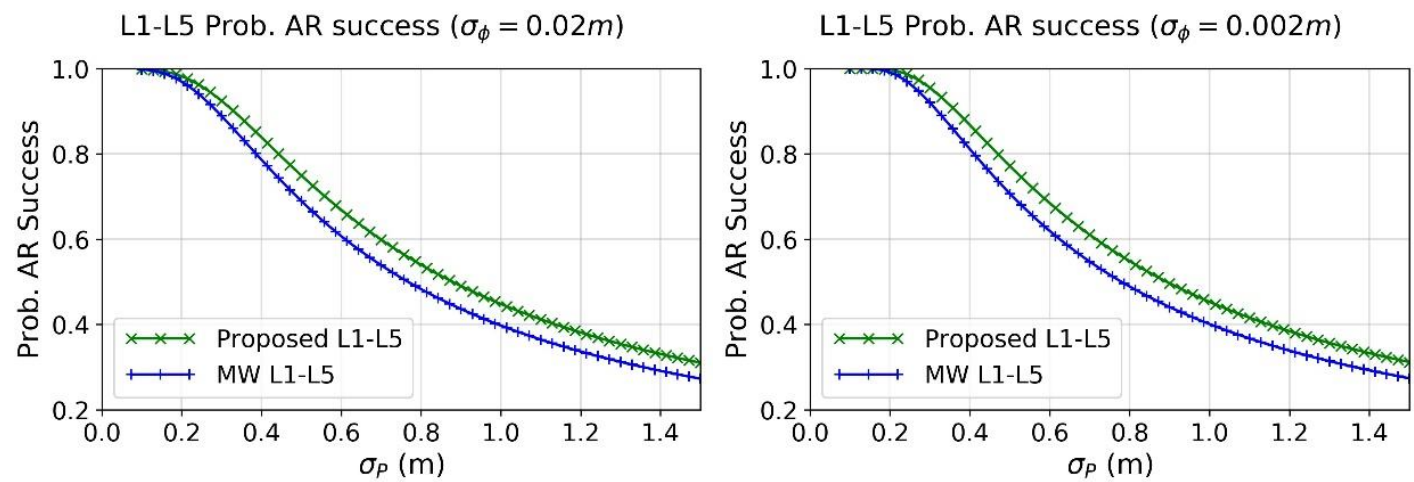

Figure 9: Probability of GPS $N_{51}$ WL integer ambiguity resolution success rate with single epoch data for the proposed model and the MW model with $\sigma_{\Phi}=0.002 \mathrm{~m}$ (left) and $\sigma_{\Phi}=0.02 \mathrm{~m}$ (right). 
Neglecting the correlation between the float WL ambiguities for the sake of simplicity, and based on error propagation law, the mean of the WL ambiguities has a std in cycles of $\sigma_{\widehat{N}_{j i}}=$ $\sigma_{N_{j i}} / \sqrt{n}$, where $n$ is the number of epochs, $\sigma_{N_{j i}}$ is the std of the population of WL ambiguities, and $\sigma_{\widehat{N}_{j i}}$ is the std for WL sample ambiguities. This relationship was used to determine the number of epochs required to achieve a Ps rate better than $99.9 \%$ for the $N_{21}, N_{51} \mathrm{WL}$ and the $N_{52}$ EWL ambiguities with $\sigma_{\mathrm{P}}(m)=[0.2,0.5,1.0,1.5,2.0,2.5]$. These range of values for $\sigma_{\mathrm{P}}$ accounts for the pseudorange measurement noise for different receiver types, and the increased multipath and noise due to tracking low elevation satellites. For example, a typical geodetic grade receiver with $\sigma_{\mathrm{P}}=0.35 \mathrm{~m}$ (El-Mowafy et al., 2015) will have an increased measurement noise of $2.0 \mathrm{~m}$ for a satellite tracked at $10^{\circ}$ elevation angle (Ele), when using a factor of 1/sin(Ele). The results, given in Table 5 and Figure 10, show that estimating the EWL ambiguity may be achieved within four epochs with $\sigma_{\mathrm{P}}=2.5 \mathrm{~m}$. For the WL ambiguities, the proposed model provides substantial benefit over the MW model, especially as the pseudorange measurement noise increases above $0.5 \mathrm{~m}$. Assuming an epoch interval of 15 seconds for uncorrelated measurements, the $N_{21}$ and $N_{51}$ WL ambiguities may be fixed within 7 minutes if $\sigma_{\mathrm{P}}<1.0 \mathrm{~m}$. One way to achieve this is by increasing the satellite cut-off elevation angle to $20^{\circ}$, which is a feasible option due to a large number of available satellites, if multiconstellation GNSS satellites are tracked. Figure 11 shows the Ps rate versus required number of uncorrelated epochs for the proposed model and MW model for the $N_{21}$ WL ambiguity. Figure 12 shows the same for the $N_{51}$ WL ambiguity. As shown, the added benefit of the proposed model is prominent for the $N_{51} \mathrm{WL}$ ambiguity fixing case with $\sigma_{\mathrm{P}}=1.5 \mathrm{~m}$.

Table 5: Number of epochs required for Ps rate of $99.9 \%$ for the $N_{21}, N_{51}$ WL and $N_{52}$ EWL ambiguities, for the proposed model and the MW model.

\begin{tabular}{|c|c|c|c|}
\hline \multirow[t]{2}{*}{ WL } & \multirow[t]{2}{*}{$\sigma_{\mathrm{P}}(\mathrm{m})$} & \multicolumn{2}{|c|}{$\begin{array}{l}\text { Number of epochs required for Ps rate of } \\
99.9 \%\end{array}$} \\
\hline & & $\begin{array}{l}\text { Proposed Linear } \\
\text { combination }\end{array}$ & $\begin{array}{l}\text { MW Linear } \\
\text { Combination }\end{array}$ \\
\hline \multirow{6}{*}{$N_{21}$} & 0.2 & 1 & 2 \\
\hline & 0.5 & 7 & 7 \\
\hline & 1.0 & 24 & 28 \\
\hline & 1.5 & 54 & 62 \\
\hline & 2.0 & 96 & 111 \\
\hline & 2.5 & 150 & 174 \\
\hline \multirow[t]{6}{*}{$N_{51}$} & 0.2 & 2 & 2 \\
\hline & 0.5 & 7 & 10 \\
\hline & 1.0 & 28 & 37 \\
\hline & 1.5 & 63 & 83 \\
\hline & 2.0 & 112 & 146 \\
\hline & 2.5 & 174 & 228 \\
\hline \multirow[t]{6}{*}{$N_{52}$} & 0.2 & 1 & 1 \\
\hline & 0.5 & 1 & 1 \\
\hline & 1.0 & 1 & 1 \\
\hline & 1.5 & 2 & 2 \\
\hline & 2.0 & 3 & 3 \\
\hline & 2.5 & 4 & 4 \\
\hline
\end{tabular}




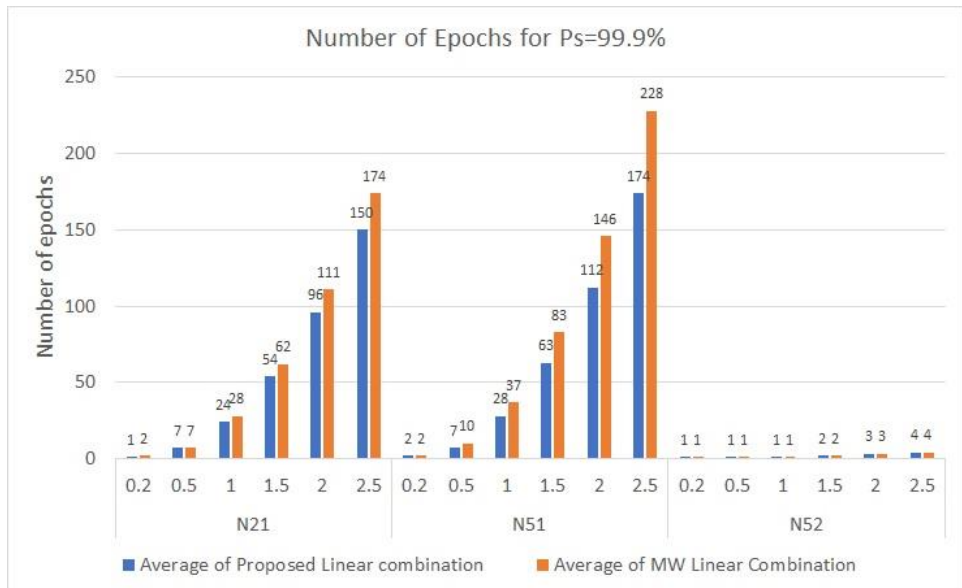

Figure 10: Number of epochs required to achieve a Ps rate of $99.9 \%$ for the $N_{21}, N_{51}$ WL and $N_{52}$ EWL ambiguities, for the proposed model and the MW model.

L1-L2 $\sigma_{\phi}=0.002 m ; \sigma_{P}=1.5 m$

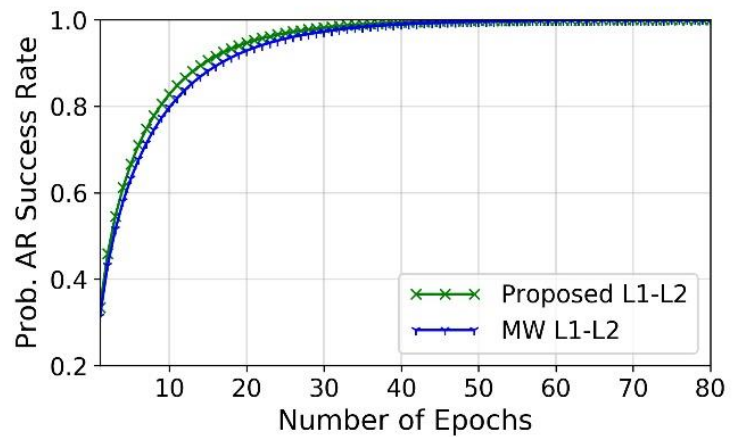

L1-L2 $\sigma_{\phi}=0.002 m ; \sigma_{P}=0.5 m$

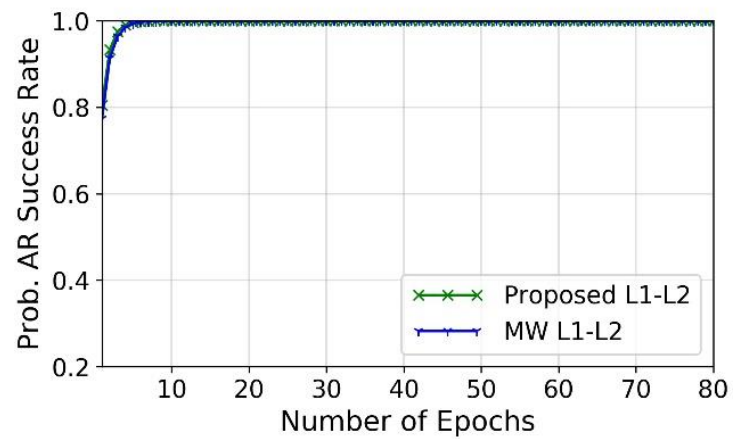

Figure 11: Probability success rate versus required number of uncorrelated epochs for the $\boldsymbol{N}_{\mathbf{2 1}}$ WL ambiguity for proposed model and MW model with $\sigma_{\mathrm{P}}=0.5 \mathrm{~m}$ (left) and $\sigma_{\mathrm{P}}=1.5 \mathrm{~m}$ (right). For both cases, $\sigma_{\Phi}=0.002 \mathrm{~m}$.
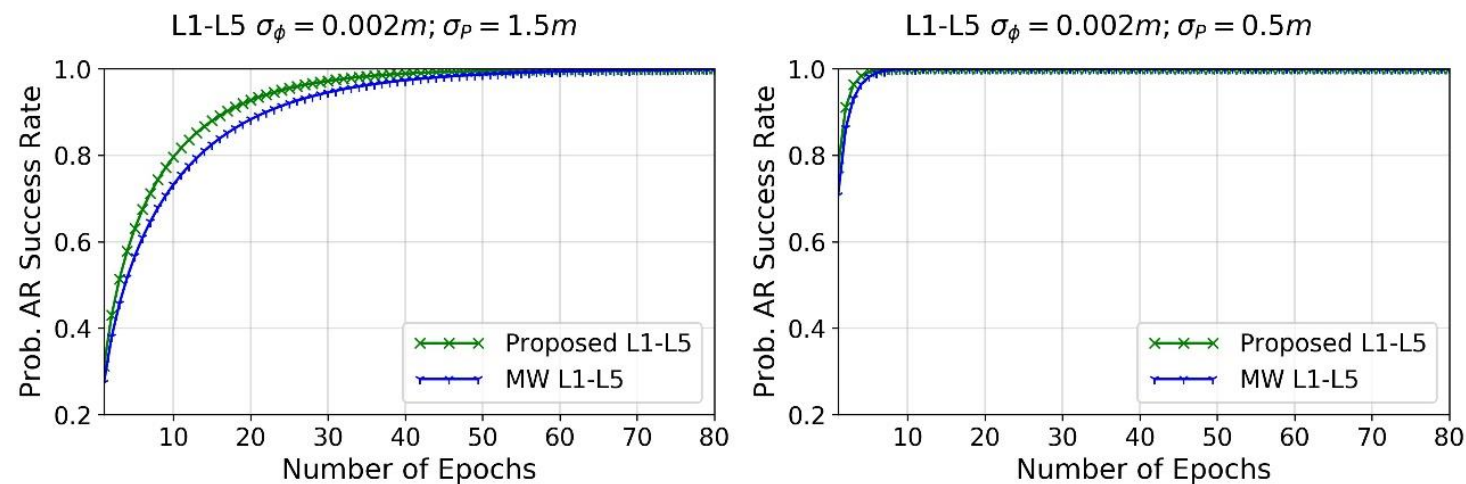

Figure 12: Probability success rate versus required number of uncorrelated epochs for the $\boldsymbol{N}_{\mathbf{5 1}} \mathrm{WL}$ ambiguity for proposed model and MW model with $\sigma_{\mathrm{P}}=0.5 \mathrm{~m}$ (left) and $\sigma_{\mathrm{P}}=1.5 \mathrm{~m}$ (right). For both cases, $\sigma_{\Phi}=0.002 \mathrm{~m}$.

\subsection{Analysis of Wide-lane Carrier Phase only Linear Combination}

The measurement noise propagation in the proposed ionosphere free combination in Section 4.1 is analysed for carrier phase std between $0.001-0.02 \mathrm{~m}$. This is compared to the noise in the triple-frequency low noise ionosphere free combination that was proposed in Li et al. (2014), also used in Laurichesse (2015). This combination was presented only for GPS and Beidou Phase II constellations by Li et al., (2014). Figure 13 shows the results for GPS, whereas Figures 14 and 15 show results for Beidou Phase II and Galileo, respectively. As shown, the measurement noise in the proposed linear combination is sensitive to the individual carrier phase measurement std. The carrier phase std must be at the mm level to retain the overall std 
in the proposed combination below $10 \mathrm{~cm}$. Such measurement precision is commonly achieved by most high-grade GNSS receivers (Geng and Bock, 2013; Groot et al., 2018).

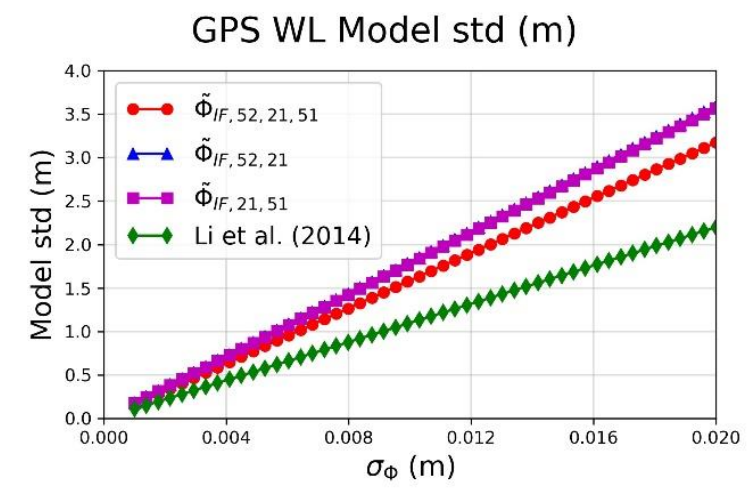

Figure 13: Comparison of measurement noise propagation in the ionosphere free combination of the three GPS carrier phase wide-lane equations $\left(\widetilde{\Phi}_{I F, 52,21,51}, \widetilde{\Phi}_{I F, 52,21}\right.$ and $\left.\widetilde{\Phi}_{I F, 21,51}\right)$ with carrier phase standard deviation between $0.001-0.02 \mathrm{~m}$.

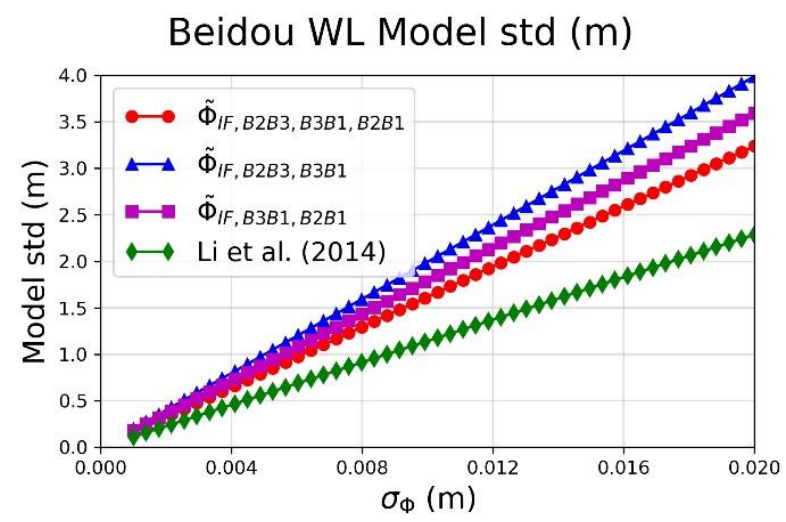

Figure 14: Comparison of measurement noise propagation in the ionosphere free combination of the three Beidou Phase II carrier phase wide-lane equations $\left(\widetilde{\Phi}_{I F, B 2 B 3, B 3 B 1, B 2 B 1} \widetilde{\Phi}_{I F, B 2 B 3, B 3 B 1}\right.$ and $\left.\widetilde{\Phi}_{I F, B 3 B 1, B 2 B 1}\right)$ with carrier phase standard deviation between 0.001-0.02m.

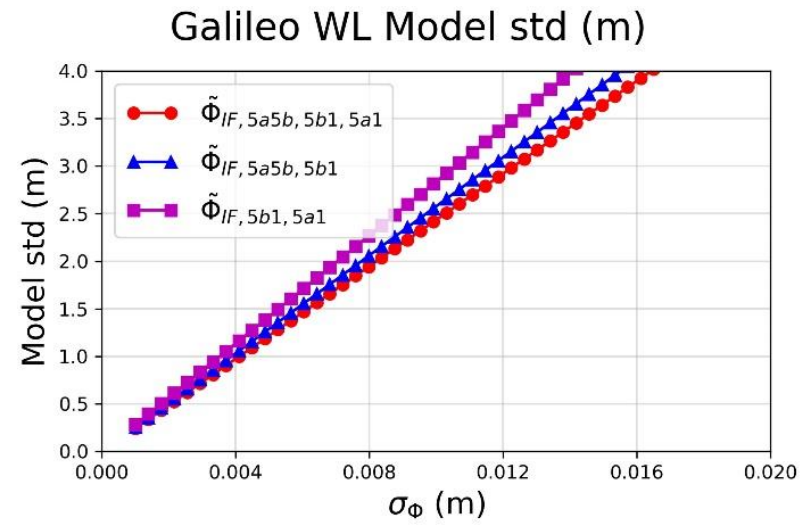

Figure 15: Comparison of measurement noise propagation in the ionosphere free combination of the three Galileo carrier phase wide-lane equations $\widetilde{(\Phi}_{I F, 5 a 5 b, 5 b 1,5 a 1}, \widetilde{\Phi}_{I F, 5 a 5 b, 5 b 1}$ and $\left.\widetilde{\Phi}_{I F, 5 b 1,5 a 1}\right)$ with carrier phase standard deviation between $0.001-0.02 \mathrm{~m}$. 


\subsection{Analysis of Two Wide-lane Carrier Phase Equations and Single Code Linear Combination}

The measurement noise propagation in the ionosphere free combination of the two-carrier phase wide-lane equations and a single pseudorange measurement proposed in Section 4.2 is analysed and compared to the combination proposed in Li et al. (2014). Figure 16 shows the overall measurement noise in the proposed linear combination for GPS with the carrier phase std in the range $0.001-0.03 \mathrm{~m}$. The left plot shows the result for pseudorange measurement std $\sigma_{P}=0.3 \mathrm{~m}$ whereas the right plot applies for $\sigma_{P}=1.0 \mathrm{~m}$. Figures 17 and 18 show the same results for Beidou II and Galileo, respectively. The overall noise in the combination proposed in Li et al. (2014), available for GPS and Beidou II only, is shown as a green line. As Figure 16 (left) shows, the overall noise for the proposed GPS $\widetilde{\Phi}_{W L, 12}, \widetilde{\Phi}_{W L, 25}$ and $P_{5}$ combination gains an improvement over the combination in Li et al. (2014) when the carrier phase noise exceeds $20 \mathrm{~mm}$. The latter method has the best performance in the typical situation when the carrier phase std is below $20 \mathrm{~mm}$. With $\sigma_{P}=1.0 \mathrm{~m}$, the combination in Li et al. (2014) maintains the best performance irrespective of the carrier phase noise. As shown in Figure 17 (left), the proposed Beidou II $\widetilde{\Phi}_{W L, B 1 B 1} \widetilde{\Phi}_{W L, B 1 B 2}$ and $P_{B 3}$ combination surpasses the performance of Li et al. (2014) when $\sigma_{\phi}>25 \mathrm{~mm}$, such as under high multipath and noise environments. When $\sigma_{P}=1.0 \mathrm{~m}$, the combination in $\mathrm{Li}$ et al. (2014) maintains the best performance irrespective of the carrier phase noise. Figure 18 (left) shows that the Galileo $\widetilde{\Phi}_{W L, E 1 E 5 a}, \widetilde{\Phi}_{W L, E 1 E 5 b}$ and $P_{E 5 a}$ combination has stable noise for $\sigma_{\phi}>10 \mathrm{~mm}$.

Since the actual pseudorange measurement is typically a large number greater than 20 thousand kilometres, the value obtained by multiplying this with the small pseudorange coefficient is significant and numerically stable. The exact values of the wide-lane phase and pseudorange coefficients calculated from Eqns. 29, 35 and 36 must be used to avoid rounding errors and loss of significance.

As Figures 16-18 show, the proposed wide-lane phase-code combination achieves improved performance over the combination of Li et al. (2014) only when the carrier phase std increases above $20 \mathrm{~mm}$ and the pseudorange std is below $0.3 \mathrm{~m}$. Such situation applies to extreme carrier phase only multipath environments. However, it is evident that the proposed method will provide similar performance to Li et al. (2014) when the carrier phase std is at 1-2mm level.
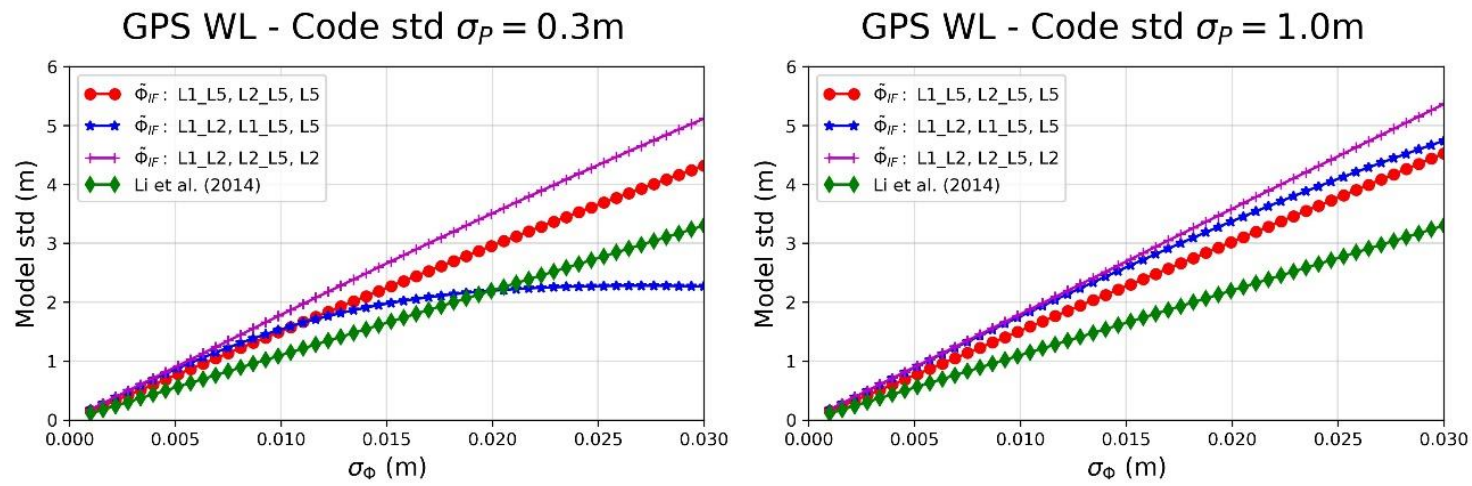

Figure 16: Overall measurement noise in the proposed two wide-lane phase and single pseudorange linear combination for GPS with the carrier phase standard deviation between $0.001 \mathrm{~m}$ to $0.03 \mathrm{~m}$ and pseudorange $\mathrm{std}=0.3 \mathrm{~m}$ (left) and pseudorange $\mathrm{std}=1.0 \mathrm{~m}$ (right). 
Beidou WL - Code std $\sigma_{P}=0.3 \mathrm{~m}$

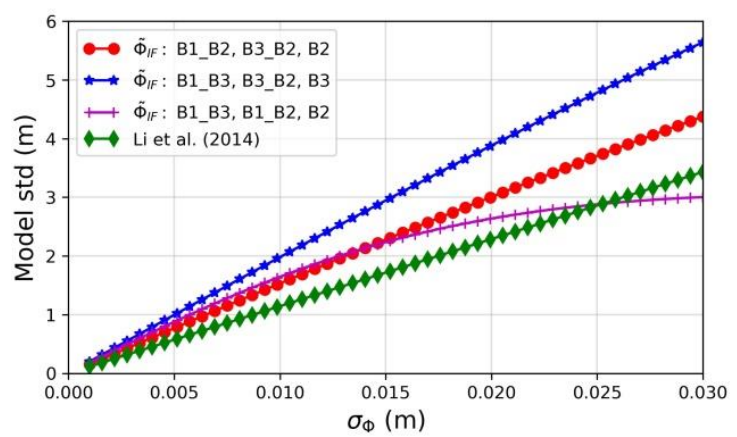

Beidou WL - Code std $\sigma_{P}=1.0 \mathrm{~m}$

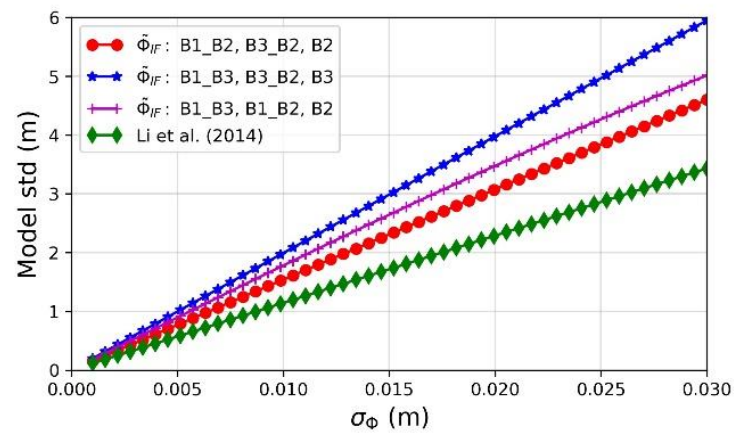

Figure 17: Overall measurement noise in the proposed two wide-lane phase and single pseudorange linear combination for Beidou Phase II with the carrier phase standard deviation between $0.001 \mathrm{~m}$ to $0.03 \mathrm{~m}$ and pseudorange $\mathrm{std}=0.3 \mathrm{~m}$ (left) and pseudorange $\mathrm{std}=1.0 \mathrm{~m}$ (right).

Galileo WL - Code std $\sigma_{P}=0.3 \mathrm{~m}$

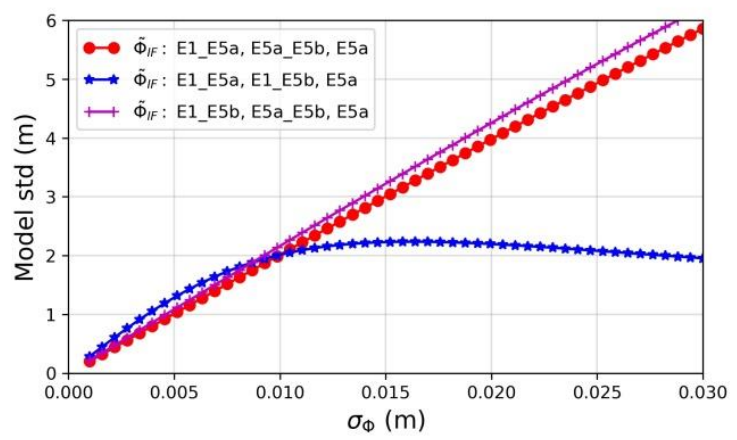

Galileo WL - Code std $\sigma_{P}=1.0 \mathrm{~m}$

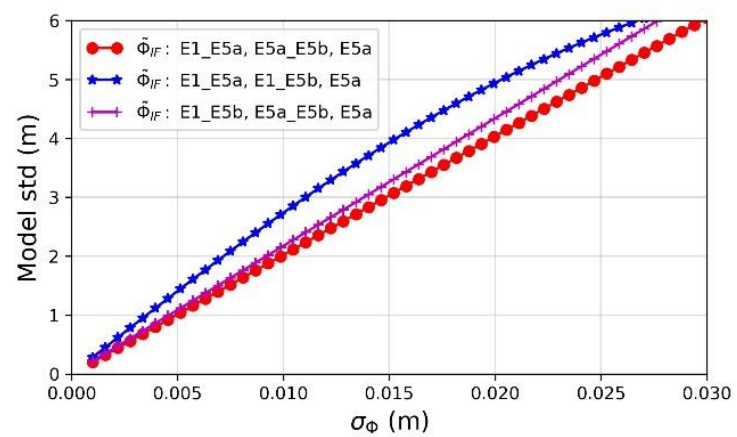

Figure 18: Overall measurement noise in the proposed two wide-lane phase and single pseudorange linear combination for Galileo with the carrier phase standard deviation between $0.001 \mathrm{~m}$ to $0.03 \mathrm{~m}$ and pseudorange $\mathrm{std}=0.3 \mathrm{~m}$ (left) and pseudorange $\mathrm{std}=1.0 \mathrm{~m}$ (right).

5.5 Analysis of Single Carrier Phase Wide-lane Equation and Two Code Linear Combination In this section, the measurement noise propagation in the ionosphere free combination of the single carrier phase wide-lane equation and pseudorange measurements on two frequencies proposed in Section 4.3 is analysed and compared to the combination proposed in $\mathrm{Li}$ et al. (2014). Figure 19 shows the overall measurement noise in the proposed linear combination for GPS with the carrier phase std in the range $0.001-0.03 \mathrm{~m}$, for a range of satellite elevation angles and under different levels of multipath effects. The left plot shows the result for pseudorange measurement std $\sigma_{P}=0.3 \mathrm{~m}$ whereas the right plot applies for $\sigma_{P}=1.0 \mathrm{~m}$. Figures 20 and 21 show the same results for Beidou II and Galileo, respectively. The overall noise in the combination proposed in $\mathrm{Li}$ et al. (2014) is shown as a green line. As Figure 19 (left) shows, the measurement noise in the proposed GPS $\widetilde{\Phi}_{W L, 25}, P_{1}$ and $P_{5}$ combination gains an improvement over the combination in Li et al. (2014) when the carrier phase noise exceeds $5 \mathrm{~mm}$. With $\sigma_{P}=1.0 \mathrm{~m}$, the proposed combination has better performance when $\sigma_{\phi}>18 \mathrm{~mm}$. As shown in Figure 20 (left), the proposed Beidou II $\widetilde{\Phi}_{W L, B 3 B 2}, P_{B 1}$ and $P_{B 2}$ combination surpasses the performance of $\mathrm{Li}$ et al. (2014) when $\sigma_{\phi}>6 \mathrm{~mm}$ and $\sigma_{P}=0.3 \mathrm{~m}$. With $\sigma_{P}=1.0 \mathrm{~m}$, the proposed combination has better performance when $\sigma_{\phi}>19 \mathrm{~mm}$. Figure 21 (left) shows that the proposed Galileo $\widetilde{\Phi}_{W L, E 5 a E 5 b}, P_{E 1}$ and $P_{E 5 a}$ combination has the best performance.

As Figures 19-20 show, the proposed single wide-lane and two pseudorange linear combination achieves improved performance over the combination of Li et al. (2014) when the carrier phase std exceeds $5-6 \mathrm{~mm}$ and the pseudorange std is below $0.3 \mathrm{~m}$. This is because the coefficients in 
the combination of $\mathrm{Li}$ at al. (2014) have large values, thus increase rapidly with an increase in carrier phase noise, whereas the pseudorange coefficients in the proposed combination have low values, thus the overall increase in noise is gradual. Even with high pseudorange std = $1.0 \mathrm{~m}$, the proposed combination gives an improvement when carrier phase std exceeds $20 \mathrm{~mm}$. This is an improved result compared to the previously analysed combination with two carrier phase wide equations a single pseudorange. Such situation applies to moderate carrier phase multipath environments with low pseudorange measurement noise. A key advantage of this method is that it only relies on the EWL ambiguities to be resolved for all the constellations, which is typically achieved with a single epoch. Thus, the user achieves decimetre level PPP without waiting for convergence.

GPS $1 \mathrm{WL}-2$ Code std $\sigma_{P}=0.3 \mathrm{~m}$

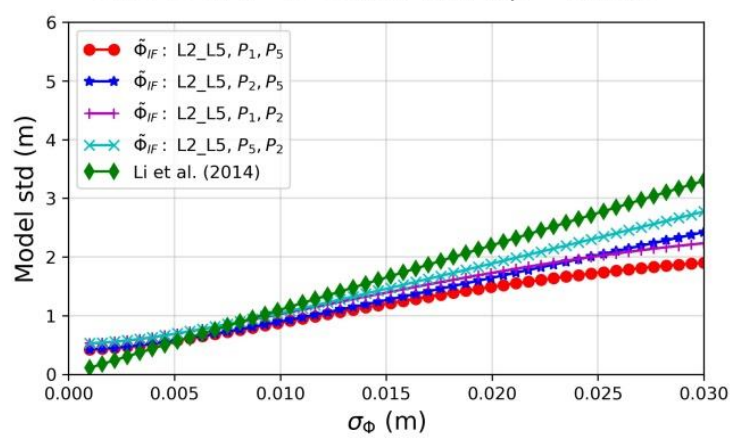

GPS $1 \mathrm{WL}-2$ Code std $\sigma_{P}=1.0 \mathrm{~m}$

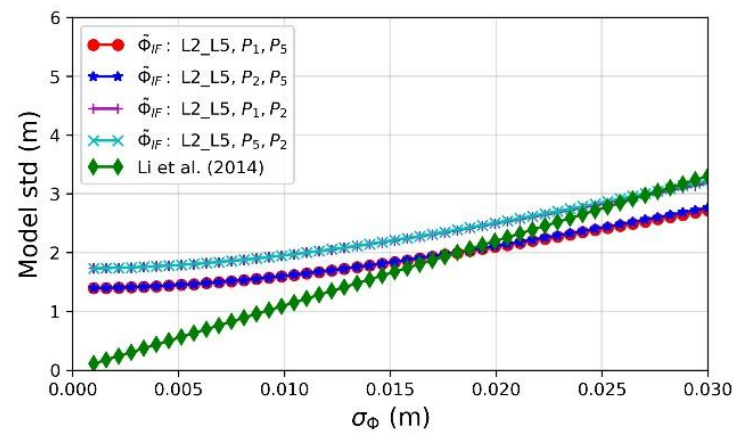

Figure 19: Overall measurement noise in the proposed linear combination for single wide-lane phase and two pseudorange measurements for GPS with the carrier phase standard deviation between $0.001 \mathrm{~m}$ to $0.03 \mathrm{~m}$ and pseudorange $\mathrm{std}=0.3 \mathrm{~m}$ (left) and pseudorange $\mathrm{std}=1.0 \mathrm{~m}$ (right).

Beidou $1 \mathrm{WL}-2$ Code std $\sigma_{P}=0.3 \mathrm{~m}$

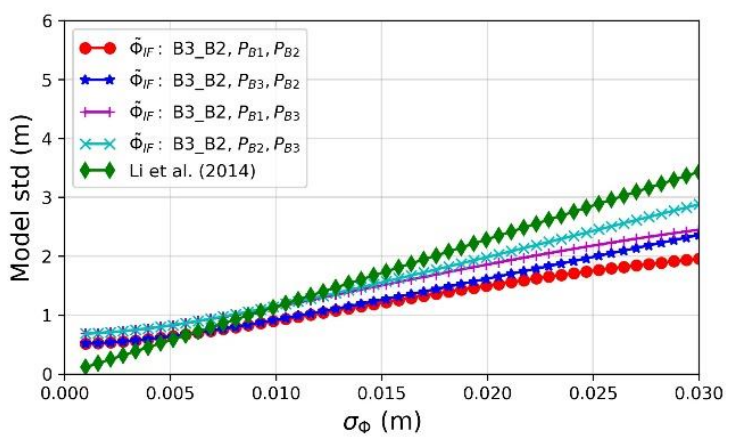

Beidou $1 \mathrm{WL}-2$ Code std $\sigma_{P}=1.0 \mathrm{~m}$

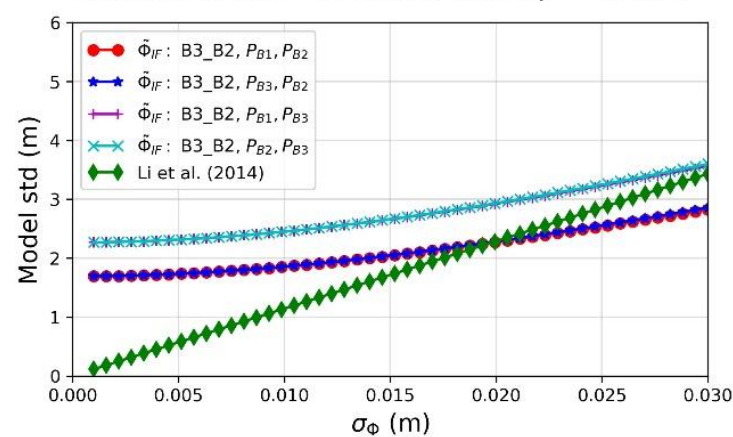

Figure 20: Overall measurement noise in the proposed linear combination for single wide-lane phase and two pseudorange measurements for Beidou Phase II with the carrier phase standard deviation between $0.001 \mathrm{~m}$ to $0.03 \mathrm{~m}$ and pseudorange $\mathrm{std}=0.3 \mathrm{~m}$ (left) and pseudorange $\mathrm{std}=1.0 \mathrm{~m}$ (right).

Galileo $1 \mathrm{WL}-2$ Code std $\sigma_{P}=0.3 \mathrm{~m}$

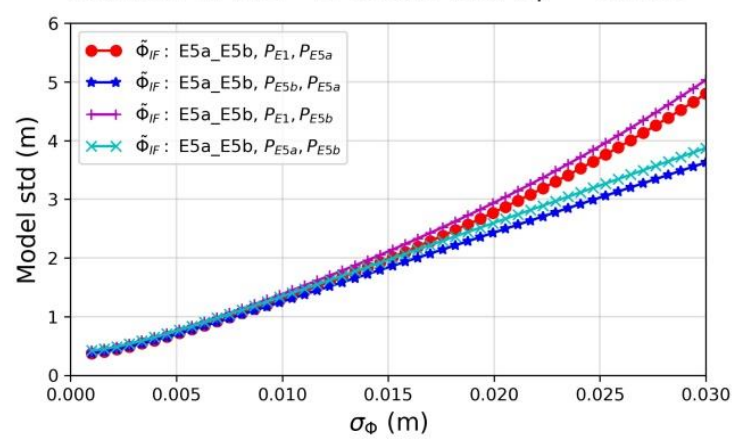

Galileo $1 \mathrm{WL}-2$ Code std $\sigma_{P}=1.0 \mathrm{~m}$

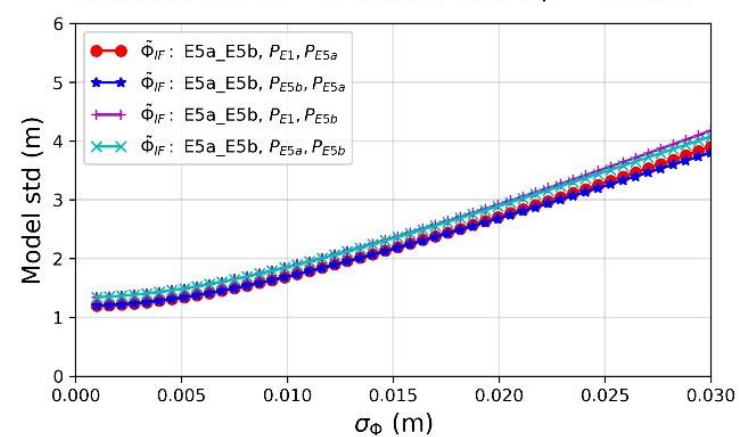


Figure 21: Overall measurement noise in the proposed linear combination for single wide-lane phase and two pseudorange measurements for Galileo with the carrier phase standard deviation between $0.001 \mathrm{~m}$ to $0.03 \mathrm{~m}$ and pseudorange $\mathrm{std}=0.3 \mathrm{~m}$ (left) and pseudorange $\mathrm{std}=1.0 \mathrm{~m}$ (right).

\subsection{Positioning Results}

This section compares the point positioning results from the proposed linear combination of a single carrier phase wide-lane equation and two code measurements with the combination of Li et al. (2014). For brevity, the GPS results are analysed firstly with carrier phase std of 0.005 cycles and pseudorange std of $0.3 \mathrm{~m}$, and secondly with carrier phase std of 0.0050 .03 cycles while maintaining pseudorange std of $0.3 \mathrm{~m}$. The analysis covers a period of 24 hours with data collected at 30 seconds interval, located at Alice Springs, Australia. The 1/ $\sin$ (elevation angle) function is used for measurement weighting. Figure 22 compares the results with the proposed combination (left) and the combination of Li et al. (2014) (right) with a $\sigma_{\phi} 0.005$ cycles. Figure 23 compares that same with an increased carrier phase std of 0.03 cycles. The results are also summarized in Table 6 . These results show that horizontal positioning accuracy of a sub 2-dm and vertical positioning accuracy of sub 5-dm is attainable with the combination of $\mathrm{Li}$ et al. (2014) if $\mathrm{mm}$ level precise carrier phase measurements are available. Here, the positioning accuracy with the proposed method is worse by a factor of 3.2. However, when the carrier phase noise is increased to 0.03 cycles, the proposed combination gives a slightly improved accuracy by a factor of 1.1 .
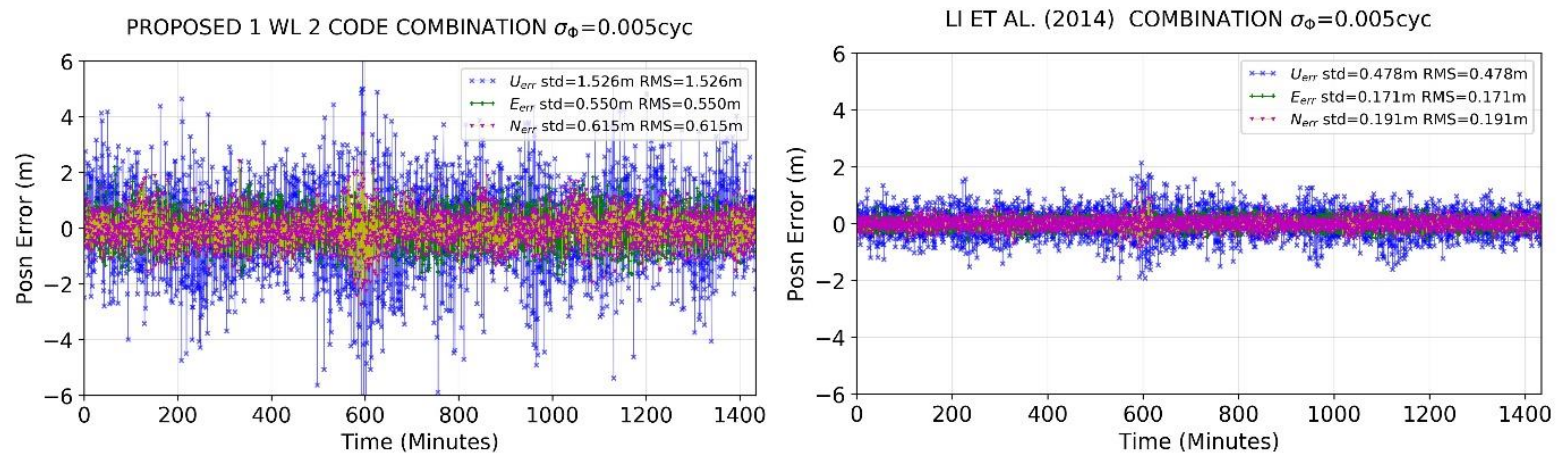

Figure 22: Positioning error in East, North and Height with the proposed linear combination of single carrier phase wide-lane and two code measurements (left) and the combination of Li et al. (2014) (right). The carrier phase std was set to 0.03 cycles and pseudorange std was $0.3 \mathrm{~m}$.
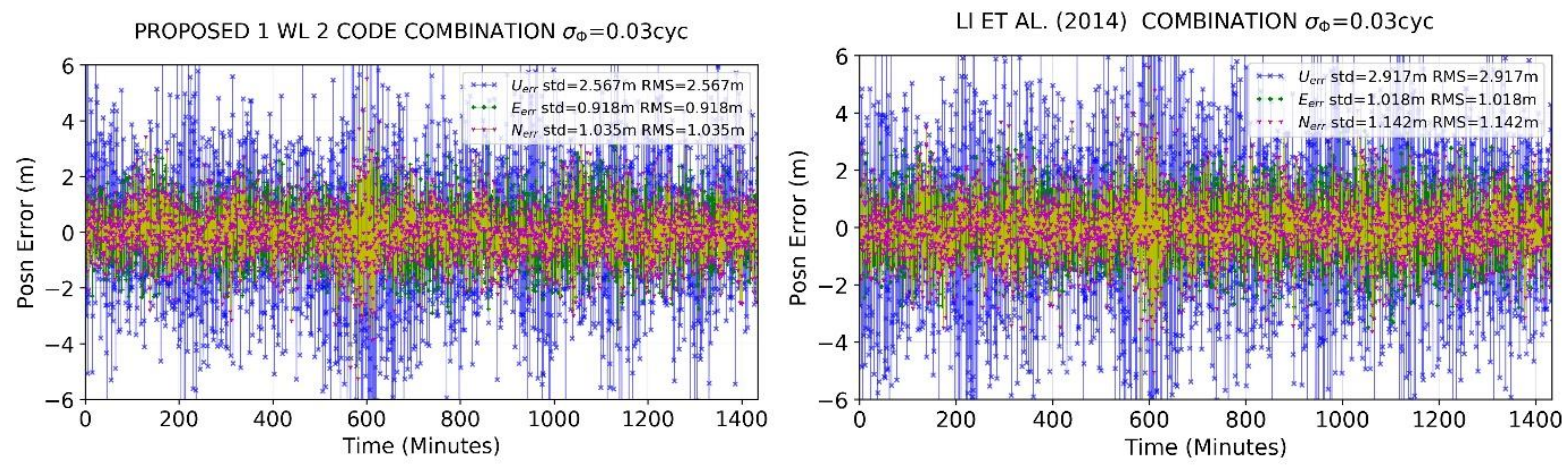

Figure 23: Positioning error in East, North and Height with the proposed linear combination of single carrier phase wide-lane and two code measurements (left) and the combination of Li et al. (2014) (right). The carrier phase std was set to 0.03 cycles and pseudorange std was $0.3 \mathrm{~m}$. 
Table 6: Comparison of point positioning performance with the the proposed linear combination of single carrier phase wide-lane and two code measurements and the combination of Li et al. (2014). The carrier phase std was set to 0.005 cycles and 0.03 cycles, respectively and pseudorange std was maintained at $0.3 \mathrm{~m}$.

\begin{tabular}{|l|l|l|l|l|c|}
\hline \multirow{2}{*}{ Component } & \multirow{2}{*}{ Model } & \multicolumn{2}{|c|}{$\sigma_{\phi}=0.005 c y c ; \sigma_{P}=0.3 m$} & \multicolumn{2}{c|}{$\sigma_{\phi}=0.03 c y c ; \sigma_{P}=0.3 m$} \\
\cline { 3 - 5 } & & Std $(\mathrm{m})$ & RMS $(\mathrm{m})$ & Std $(\mathrm{m})$ & RMS $(\mathrm{m})$ \\
\hline \multirow{2}{*}{ East } & Proposed & 0.550 & 0.550 & 0.918 & 0.918 \\
\cline { 2 - 5 } & Li et al. (2014) & 0.171 & 1.171 & 1.018 & 1.018 \\
\hline \multirow{2}{*}{ North } & Proposed & 0.615 & 0.615 & 1.035 & 1.035 \\
\cline { 2 - 5 } & Li et al. (2014) & 0.191 & 0.191 & 1.142 & 1.142 \\
\hline \multirow{2}{*}{ Height } & Proposed & 1.526 & 1.526 & 2.567 & 2.567 \\
\cline { 2 - 5 } & Li et al. (2014) & 0.478 & 0.478 & 2.917 & 2.917 \\
\hline
\end{tabular}

\section{Conclusion}

In this paper, we firstly presented an improved linear combination for estimating the EWL/WL ambiguities. All EWL ambiguities were fixed with one epoch for both methods. For the proposed method, all the EWL and WL ambiguities were fixed in less than two minutes. This shows that use of the proposed WL linear combination enables faster estimation of the WL ambiguities compared to the MW method. Thus, the user achieves decimetre level PPP without waiting for the traditional 30 minutes on average convergence-time. Compared to the MW linear combination for the GPS case, the measurement noise in the proposed combination was $6.7 \%$ lower for L1/L2, $12.7 \%$ lower for L1/L5 and a modest $0.7 \%$ lower for the L2/L5. Data analysis at four sites showed that the $\mathrm{N}_{21}$ WL ambiguities were fixed correctly with a single epoch for $88.9 \%$ of cases, an improvement over the MW method by $5.4 \%$. The $\mathrm{N}_{51} \mathrm{WL}$ ambiguities were fixed correctly with one epoch for $86.0 \%$ of cases, an improvement over the MW method by $5.9 \%$.

To fix the $N_{21}$ WL ambiguity with a single epoch with Ps $=99.9 \%$ requires that $\sigma_{\mathrm{P}} \leq 0.18 \mathrm{~m}$, which is possible with the modernised signals of the GNSS systems. Fixing the $N_{51} \mathrm{WL}$ ambiguity with a single epoch with the same Ps would require that $\sigma_{\mathrm{P}} \leq 0.10 \mathrm{~m}$. The number of uncorrelated epochs required to fix the EWL/ WL ambiguity with Ps=99.9\% was also analysed. The EWL ambiguity may be fixed with just four epochs of data with $\sigma_{\mathrm{P}}$ as high as $2.5 \mathrm{~m}$. Although the proposed model provides substantial benefit over the MW model for fixing the WL ambiguities, fixing the $N_{21}$ and $N_{51}$ WL ambiguities within 7 minutes (assuming an epoch interval of 15 seconds) requires that $\sigma_{\mathrm{P}}<1.0 \mathrm{~m}$. One way to achieve this is by increasing the satellite cut-off elevation angle to $20^{\circ}$. This is a feasible option due to a large number of available satellites, if multi-constellation GNSS satellites are tracked by the receiver.

After successfully fixing the integer EWL and WL ambiguities with the newly proposed linear combinations, three methods were proposed to enable PPP at dm-level positioning accuracy. The first model used the three EWL/ WL ambiguities in their respective carrier phase only WL equations to derive an IF linear combination with lowest measurement noise propagation. This method is capable of providing decimetre level accuracy if $\mathrm{mm}$ level precise carrier phase measurements are available, which is achievable by high-grade geodetic receivers. The second model used two of the EWL/ WL carrier phase only equations and a single pseudorange measurement to derive a linear combination with the same properties. This method gave superior performance than the method of Li et al. (2014) in extreme carrier phase multipath conditions when $\sigma_{\Phi}>20 \mathrm{~mm}$. The third model used a single WL carrier phase equation and pseudorange measurements on two frequencies to derive an IF linear combination with least noise propagation. The performance of this method exceeds that of Li et al. (2014) only when $\sigma_{\Phi}>5 \mathrm{~mm}$ and $\sigma_{P}$ is maintained at $0.3 \mathrm{~m}$. 


\section{References}

1. Bisnath S and Gao Y (2009) Precise Point Positioning: A powerful Technique with a Promising Future, GPS World, April 2009, http://www2.unb.ca/gge/Resources/gpsworld.april09.pdf.

2. Bisnath S, Aggrey J, Seepersad G and Gill Maninder (2018) Examining precise point positioning now and in the future, GPS World, March 2019, https://www.gpsworld.com/innovation-examining-precise-point-positioning-now-and-inthe-future.

3. Cocard M, Bourgon S, Kamali O and Collins P (2008) A systematic investigation of optimal carrier-phase combinations for modernized triple-frequency GPS, Journal of Geodesy, 82: 555-564, DOI: 10.1007/s00190-007-201-x.

4. Collins P, Bisnath S, Lahaye F and Heroux P (2010). Undifferenced GPS Ambiguity Resolution Using the Decoupled Clock Model and Ambiguity Datum Fixing. Navigation, 57(2): $123-135$.

5. Deo M and El-Mowafy A (2016) Triple-frequency GNSS models for PPP with float ambiguity estimation: performance comparison using GPS, Survey Review, Published online 3 Dec 2016, https://doi.org/10.1080/00396265.2016.1263179.

6. El-Mowafy A (2014) GNSS Multi-Frequency Receiver Single-Satellite Measurement Validation Method, GPS Solutions 18(4): 553-561, doi: 10.1007/s10291-013-0352-6.

7. El-Mowafy A (2015) Estimation of Multi-Constellation GNSS Observation Stochastic Properties Using a Single-Receiver Single-Satellite Data Validation Method, Survey Review, 47(341): 99-108.

8. El-Mowafy A, Deo M and Rizos C (2016) On Biases in Precise Point Positioning with Multi-Constellation and Multi-Frequency GNSS Data. Measurement Science and Technology, 27(3), 035102.

9. Ge M, Gendt G, Rothacher M, Shi C and Liu J (2008) Resolution of GPS carrier-phase ambiguities in precise point positioning (PPP) with daily observations, Journal of Geodesy 82(7):389-399, doi:10.1007/s00190-007-0187-4.

10. Geng J and Bock Y (2013) Triple-frequency GPS precise point positioning with rapid ambiguity resolution, Journal of Geodesy, 87: 449-460.

11. Geng J, Meng X, Dodson AH and Teferle F (2010) Integer ambiguity resolution in Precise Point Positioning: Method comparison, Journal of Geodesy 84(9): 569-581.

12. Groot L, Infante E, Jokinen A, Kruger B and Norman L (2018) Precise Positioning for Automotive with Mass Market GNSS Chipsets, 31st International Technical Meeting of the Satellite Division of the Institute of Navigation (ION GNSS+ 2018), Miami, Florida, September 24-28.

13. Héroux P and Kouba J (2001) GPS precise point positioning using IGS orbit products, Physics and Chemistry of the Earth, Part A: Solid Earth and Geodesy, 26(6-8): 573-578, https://doi.org/10.1016/S1464-1895(01)00103-X.

14. Jung J, Enge P and Pervan B (2000) Optimization of cascade integer resolution with three civil GPS frequencies. In: Proceedings of ION GPS 13th international technical meeting of the satellite division, Salt Lake City, UT, pp 2191-2200.

15. Laurichesse D (2015) Handling the Biases for Improved Triple-Frequency PPP Convergence, GPS World, 26(4), April 2015.

16. Laurichesse D, Mercier F, Berthais JP, Broca P and Cerri L (2009) Integer ambiguity resolution on undifferenced GPS phase measurements and its application to PPP and satellite precise orbit determination, Navigation 56(2): 135-149. 
17. Li B (2018) Review of triple-frequency GNSS: ambiguity resolution, benefits and challenges, The Journal of Global Positioning Systems (2018) 16:1, DOI 10.1186/s41445018-0010-y.

18. Li B, Feng Y, Shen Y (2010) Three carrier ambiguity resolution: distance independent performance demonstrated using semi-generated triple frequency GPS signals. GPS Solutions 14(2): 177-184.

19. Li T, Wang J and Laurichesse D (2014) Modelling and quality control for reliable precise point positioning integer ambiguity resolution with GNSS modernization, GPS Solutions (2014) 18:429-442, DOI 10.1007/s10291-013-0342-8.

20. Teunissen PJG (1998) Success probability of integer GPS ambiguity rounding and bootstrapping. Journal of Geodesy 72(10):606-612.

21. Urquhart L (2009) An analysis of multi-frequency carrier phase linear combinations for GNSS, Senior technical report, Department of Geodesy and Geomatics Engineering Technical Report No. 263, University of New Brunswick, Fredericton, New Brunswick, Canada, 71 pp.

22. Vollath U, Birnbach S and Landau H (1998) Analysis of three carrier ambiguity resolution (TCAR) technique for precise relative positioning in GNSS-2. ION GPS 1998:417-426.

23. Zumberge, JF, Heflin, MB Jefferson, DC, Watkins, MM and Webb FH (1997) Precise Point Positioning for the Efficient and Robust Analysis of GPS Data from Large Networks, Journal of Geophysical Research, 102(B3): 5005-5017. 